\title{
Vector boson pair production at the LHC
}

\section{John M. Campbell, R. Keith Ellis and Ciaran Williams}

Fermilab,

Batavia, IL 60510, U.S.A.

E-mail: johnmc@fnal.gov, ellis@fnal.gov, ciaran@fnal.gov

ABSTRACT: We present phenomenological results for vector boson pair production at the LHC, obtained using the parton-level next-to-leading order program MCFM. We include the implementation of a new process in the code, $p p \rightarrow \gamma \gamma$, and important updates to existing processes. We incorporate fragmentation contributions in order to allow for the experimental isolation of photons in $\gamma \gamma, W \gamma$, and $Z \gamma$ production and also account for gluon-gluon initial state contributions for all relevant processes. We present results for a variety of phenomenological scenarios, at the current operating energy of $\sqrt{s}=7 \mathrm{TeV}$ and for the ultimate machine goal, $\sqrt{s}=14 \mathrm{TeV}$. We investigate the impact of our predictions on several important distributions that enter into searches for new physics at the LHC.

KeYwords: QCD Phenomenology

ARXIV EPRINT: 1105.0020 


\section{Contents}

1 Introduction $\quad 2$

2 Photon fragmentation 3

3 Overview 5

$4 \quad \gamma \gamma$ production $\quad 6$

4.1 Description of the calculation 6

$\begin{array}{lll}4.2 & \text { Results } & 7\end{array}$

$\begin{array}{lll}5 & W^{ \pm} \gamma \text { production } & 11\end{array}$

$\begin{array}{ll}5.1 \text { Description of the calculation } & 11\end{array}$

$\begin{array}{lll}5.2 & \text { Results } & 12\end{array}$

$6 \quad Z_{\gamma}$ production $\quad \mathbf{1 5}$

$\begin{array}{ll}\text { 6.1 Description of the calculation } & 15\end{array}$

$\begin{array}{lll}6.2 \text { Results } & 16\end{array}$

$\begin{array}{lll}7 & W W \text { production } & 18\end{array}$

$\begin{array}{ll}7.1 & \text { Description of the calculation } \\ \end{array}$

$\begin{array}{lll}7.2 & \text { Results } & 19\end{array}$

$8 W^{ \pm} Z$ production $\quad 21$

8.1 Description of the calculation 21

8.2 Results 22

$9 \quad Z Z$ production $\quad 24$

9.1 Description of the calculation 24

9.2 Results 24

10 Conclusions $\quad 25$

$\begin{array}{ll}\text { A Input parameters for phenomenological results } & \mathbf{2 8}\end{array}$

B Helicity amplitudes for gluon-gluon processes 28

B.1 Notation 28

B.2 Amplitudes for $g g \rightarrow Z \gamma \quad 29$

B.3 Amplitudes for $g g \rightarrow W W \quad 31$

B.4 Amplitudes for $g g \rightarrow Z Z \quad 31$ 


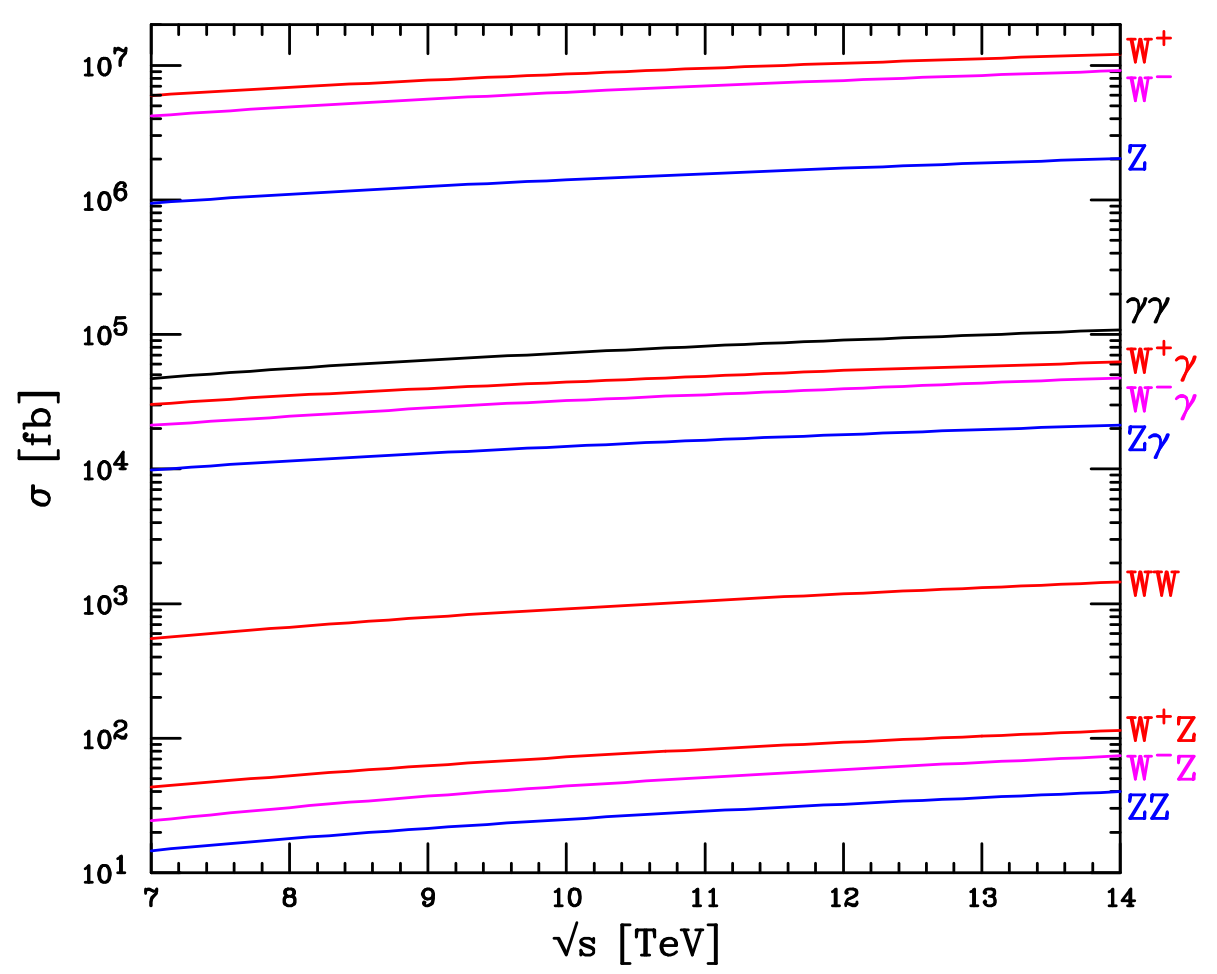

Figure 1. NLO boson production in $p p$-collisions. The decay branching ratios of the $W$ 's and $Z$ 's into one species of leptons are included. For $\gamma \gamma$ and $V \gamma$ we apply $p_{T}$ cuts of 25 and $10 \mathrm{GeV}$ to photons respectively.

\section{Introduction}

The current plan for the LHC calls for running in both 2011 and 2012. Running in 2011 is at a centre of mass energy at $\sqrt{s}=7 \mathrm{TeV}$, with a baseline expectation of $1 \mathrm{fb}^{-1}$ per experiment and a good chance that greater luminosity will be accumulated. At the end of the 2012 run it is likely that data samples in excess of $5 \mathrm{fb}^{-1}$ will have been accumulated by both of the general purpose detectors. Data samples of this size will (at the very least) allow detailed studies of the production of pairs of vector bosons.

It therefore seems opportune to provide up-to-date predictions for the production of all pairs of vector bosons, specifically for the LHC operating at $7 \mathrm{TeV}$. This extends the previous implementation of diboson production in MCFM [1] which was focussed primarily on the Tevatron. Moreover, we also consider the production of final states that contain real photons. This requires the inclusion of fragmentation contributions in order to address the issue of isolation in an experimental context. In addition, we have also included the contribution of the gluon-gluon initial state to a number of processes. These finite corrections are formally of higher order but can be of phenomenological relevance at the LHC where the gluon flux is substantial.

A review of the current experimental status of vector pair boson production, primarily from the Tevatron, can be found in ref. [2]. The production of pairs of vector bosons is crucial both in order to check the gauge structure of the Standard Model (SM) and in the 
search for new physics. This is because production of vector boson pairs and the associated particles from their decay, enter as irreducible backgrounds for many Higgs and new physics searches. The observationally most promising decays of the Higgs boson are to two photons (for a light Higgs), or to two $W$ 's or two $Z$ 's that decay leptonically. Clearly vector boson pair production is an irreducible background in these searches. Processes with leptons and missing energy are typical signatures of many new physics models, of which supersymmetry is a classic example. Again, knowledge of SM processes which possess multiple leptons and missing energy is crucial in the quest to discover or rule out these models.

In figure 1 we show the rates for various electroweak processes at energies between $\sqrt{s}=7$ and $14 \mathrm{TeV}$. This figure serves both as a road-map to this paper and as an indication of the relative size of the various diboson processes. We present the cross sections for single boson production to illustrate the orders of magnitude which separate single boson and diboson production. Where appropriate we have included the branching ratios of vector bosons to a single family of leptons and applied a transverse momentum cut of $10 \mathrm{GeV}$ $(W \gamma$ and $Z \gamma)$ and $25 \mathrm{GeV}(\gamma \gamma)$ to photons. No other cuts are applied to the boson decay products.

Updating the diboson processes in MCFM for the new energy range probed at the LHC is the primary aim of this work. With that in mind we begin in section 2 by outlining the steps needed to include photon fragmentation in the code. Section 3 serves as an overview, describing the parameters that we use and outlining the processes that receive extra corrections from gluon initiated production mechanisms. Section 4 discusses the phenomenology of $\gamma \gamma$ production at the LHC. We investigate the role of isolation on the cross section and the impact of Higgs search cuts on di-photon production. Sections 5 and 6 contain our predictions for $W \gamma$ and $Z \gamma$ production at the LHC. We investigate the role of final-state radiation in our calculations and compare our NLO results with the recently reported cross sections from CMS [3]. Sections 7, 8 and 9 turn to the production of two massive vector bosons. We are able to compare our prediction for the $W W$ cross section with early results from ATLAS and CMS $[4,5]$. We examine the effect of the gluon initiated processes in the $W W$ and $Z Z$ final states, with particular emphasis on their role as Higgs backgrounds. For $W Z$ production we discuss briefly the properties of boosted $Z$ 's. Finally in section 10 we draw our conclusions. Appendix A contains a more detailed discussion of our electroweak parameters whilst appendix B presents formulae for the $g g \rightarrow V_{1} V_{2}$ amplitudes as implemented in MCFM.

\section{Photon fragmentation}

Since we will consider a number of final states including photons we must first discuss the additional complications that this involves, compared to the production of $W$ and $Z$ bosons. Experimentally, the production of photons occurs via two mechanisms. Prompt photons are produced in hard scattering processes whilst secondary photons arise from the decays of particles such as the $\pi^{0}$. Since secondary photons are typically associated with hadronic activity one can attempt to separate these contributions by limiting the amount of hadronic energy in a cone of size $R_{0}=\sqrt{ }\left(\Delta \eta^{2}+\Delta \phi^{2}\right)$ around the photon. Experimental 
isolation cuts are of the form,

$$
\sum_{\in R_{0}} E_{T}(\mathrm{had})<\epsilon_{h} p_{T}^{\gamma} \quad \text { or } \quad \sum_{\in R_{0}} E_{T}(\mathrm{had})<E_{T}^{\max } .
$$

Thus the transverse hadronic energy, $E_{T}$ (had), is limited to be some small fraction of the transverse momentum of the photon or cut off at a fixed, small upper limit.

Matters are complicated both experimentally and theoretically by a second source of prompt photons. A hard QCD parton can fragment non-perturbatively into a photon. As a result a typical photon production cross section takes the form,

$$
\sigma=\sigma^{\gamma}\left(M_{F}^{2}\right)+\int d z D^{a}(z) \sigma^{a}\left(z, M_{F}^{2}\right) .
$$

Here $\sigma^{\gamma}$ represents the direct component of the photon production cross section whilst the second term arises from the fragmentation of a parton $a$ into a photon with momentum $z p^{a}$. Each contribution separately depends on the fragmentation scale, $M_{F}$. The fragmentation functions, taken as solutions to a DGLAP equation are of (leading) order $\alpha_{E W} / \alpha_{s}$. This means that they are formally of the same order as the leading order direct term. At highenergy hadron colliders, the QCD tree-level matrix element, coupled to a fragmentation function can become the dominant source of prompt photon production. However, the magnitude of these terms can be drastically reduced by applying the isolation cuts described above. This is due to the fact that the fragmentation functions strongly favour the low $z$ region. Once the photon is isolated, $z$ is typically large enough that the fragmentation contribution drops substantially from the unisolated case.

A theoretical description of isolated photons is complicated because of the occurence of collinear singularities between photons and final-state quarks. A finite cross section is only obtained when these singularities are absorbed into the fragmentation functions. As a result the only theoretically well-defined NLO quantity is the sum of the direct and fragmentation contributions. Once these two contributions are included one can isolate the photon using the cuts of eq. (2.1) in an infrared safe way [6].

Although the underlying dynamics of photon fragmentation are non-perturbative the evolution of the functions with the scale $M_{F}$ is perturbative. In the same manner as the parton distribution functions, the fragmentation functions satisfy a DGLAP evolution equation. In MCFM we use the fragmentation functions of ref. [7], which are NLL solutions to the DGLAP equation.

Final state quark-photon collinear singularities are removed using a variant [6] of the Catani-Seymour dipole subtraction formalism [8]. More specifically, we treat the photon in the same manner as one would treat an identified final state parton (with the appropriate change of colour and coupling factors). Integration of these subtraction terms over the additional parton phase space yields pole pieces of the form [6],

$$
D_{q}^{\gamma}=-\frac{1}{\epsilon} \frac{\Gamma(1-\epsilon)}{\Gamma(1-2 \epsilon)}\left(\frac{4 \pi \mu^{2}}{M_{F}^{2}}\right) \frac{\alpha}{2 \pi} e_{q}^{2} P_{\gamma q}(z)
$$

where $P_{\gamma q}(z)$ is the tree level photon-quark splitting function. This piece $D_{q}^{\gamma}$ is the lowest order definition of the photon fragmentation function in the $\overline{M S}$ scheme. This singularity is then absorbed into the fragmentation functions to yield finite cross sections. 
Since the isolation cuts reduce the magnitude of the fragmentation contributions we calculate the QCD matrix elements $\sigma^{a}\left(z, M_{F}^{2}\right)$ to LO, i.e. we neglect NLO corrections to the fragmentation processes.

An alternative procedure, in which one can avoid calculating the fragmentation contributions altogether, is to follow the smooth cone isolation of Frixione [9]. In such an approach one applies the following isolation prescription to the photon,

$$
\sum_{R_{j \gamma} \in R_{0}} E_{T}(\mathrm{had})<\epsilon_{h} p_{T}^{\gamma}\left(\frac{1-\cos R_{j \gamma}}{1-\cos R_{0}}\right) .
$$

Using this prescription, soft radiation is allowed inside the photon cone but collinear singularities are removed. Since the smooth-cone isolation is infra-red finite, there is no need to include fragmentation contributions in this case. Currently this isolation is difficult to implement experimentally and therefore it is not used in this paper. ${ }^{1}$

\section{Overview}

The results presented in this paper are obtained with the latest version of the MCFM code (v6.0). We use the default set of electroweak parameters as described in appendix A.

For the parton distribution functions (pdfs) we use the sets of Martin, Stirling, Thorne and Watt [10]. For the calculation of the LO results presented here we employ the corresponding LO pdf fit, with 1-loop running of the strong coupling and $\alpha_{s}\left(M_{Z}\right)=0.13939$. Similarly, at NLO we use the NLO pdf fit, with $\alpha_{s}\left(M_{Z}\right)=0.12018$ and 2-loop running. The fragmentation of partons into photons uses the parametrization "set II" of Bourhis, Fontannaz and Guillet [7].

As mentioned in the introduction, for several processes we have included contributions of the form $g g \rightarrow V_{1} V_{2}$. These contributions proceed through a closed fermion loop and form a gauge invariant subset of the one-loop amplitudes. However, since there is no $g g$ tree level contribution the first time these pieces enter in the perturbative expansion is at $\alpha_{S}^{2}$, (i.e. NNLO). Simple power counting would thus lead one to assume that these pieces are small, of the order of a few percent of the LO cross section. At the LHC this is often not the case, since the large gluon flux in the pdfs can overcome the $\mathcal{O}\left(\alpha_{S}^{2}\right)$ suppression in the perturbative expansion. The resulting gluon-gluon contributions are instead $\mathcal{O}(10 \%)$ of the LO cross section, i.e. these pieces are comparable to the other NLO contributions.

Charge conservation ensures that not all diboson processes receive these gluon-gluon initiated contributions. The allowed processes are $g g \rightarrow\left\{\gamma \gamma, Z \gamma, W^{+} W^{-}, Z Z\right\}$, each of which has been studied in some format in the past [11-26]. We refer the reader to the appropriate section for details of each calculation. We note that for $g g \rightarrow\left\{Z \gamma, W^{+} W^{-}, Z Z\right\} \rightarrow$ leptons, we present (to the best of our knowledge) analytic formulae for the helicity amplitudes for the first time. These formulae were readily obtained from the amplitudes for the process $e^{+} e^{-} \rightarrow 4$ partons [27].

Since there are no $g g$ tree level contributions, each of these 1-loop amplitudes is both infrared and ultraviolet finite. This means that, once calculated, these contributions are

\footnotetext{
${ }^{1}$ Smooth cone isolation is however available in MCFM for theoretical comparisons.
} 
simple to implement in MCFM. Throughout this paper we include these pieces in the NLO results except for the $g g \rightarrow \gamma \gamma$ section. Since the strong corrections to the $g g \rightarrow \gamma \gamma$ process, including the two-loop amplitude [13] are known and sizeable [14], we proceed differently for this process. The two-loop $g g \rightarrow \gamma \gamma$ amplitude is infrared divergent and must be combined with real radiative corrections in exactly the same manner as a canonical NLO contribution [14]. As a result these contributions are included in our NLO predictions for the diphoton process, while the one-loop $g g \rightarrow \gamma \gamma$ calculation is included in the LO result.

\section{$4 \gamma \gamma$ production}

\subsection{Description of the calculation}

In view of its role as the principal background in the search for the light Higgs boson in the decay mode $H \rightarrow \gamma \gamma$, it is important that the prediction for Standard Model diphoton production is as accurate as possible. The production of photons in hadronhadron interactions proceeds through the Born level process,

$$
q+\bar{q} \rightarrow \gamma \gamma
$$

Corrections to this picture due to QCD interactions have been first considered at $O\left(\alpha_{s}\right)$ in ref. [28] and the results for that process have been included in the Diphox Monte Carlo [29]. The large flux of gluons at high energy - in particular at current LHC energies - means that diagrams involving loops of quarks can give a significant additional contribution [11, $12,15]$,

$$
g+g \rightarrow \gamma \gamma
$$

Since these contributions can be rather large, in order to obtain a reliable estimate of their contribution to the diphoton cross section it is necessary to include higher order corrections. The results of such a calculation, involving two-loop virtual contributions [13], were presented in ref. [14].

The results presented in this section are obtained using our current implementation in MCFM which is as follows. The $g g$ process is included at NLO using the two loop matrix elements of ref. [13] and following the implementation of ref. [14]. We include five flavours of massless quarks and neglect the effect of the top quark loops, which are suppressed by $1 / m_{t}^{4}$. Next-to-leading order corrections to the $q \bar{q}$ initiated process are more straightforward to include, although some care is required due to the issues of photon fragmentation and isolation that have been described in section 2 .

We can compare our implementation of $p p \rightarrow \gamma \gamma$ to Diphox [29]. Diphox contains NLO predictions for both the direct and fragmentation pieces, but only includes the $g g$ initiated pieces at leading order. In MCFM we include NLO predictions for the direct pieces, LO predictions for the fragmentation processes (using NLL fragmentation functions) and the "NLO" $g g$ predictions. For isolated photons the "NLO" $g g$ corrections represent around $5 \%$ of the total cross section, so we expect them to be at least as important as the NLO corrections to the fragmentation piece. 


\begin{tabular}{|c|c|c|}
\hline$\sqrt{s}[\mathrm{TeV}]$ & $\sigma^{L O}(\gamma \gamma)[\mathrm{pb}]$ & $\sigma^{N L O}(\gamma \gamma)[\mathrm{pb}]$ \\
\hline 7 & $35.98(0)$ & $47.0(1)_{-6 \%}^{+5 \%}$ \\
8 & $43.04(1)$ & $55.8(1)_{-6 \%}^{+4 \%}$ \\
9 & $50.32(1)$ & $64.3(1)_{-5 \%}^{+5 \%}$ \\
10 & $57.76(1)$ & $73.0(2)_{-5 \%}^{+4 \%}$ \\
11 & $65.37(1)$ & $81.8(2)_{-5 \%}^{+3 \%}$ \\
12 & $73.07(1)$ & $90.5(3)_{-5 \%}^{+4 \%}$ \\
13 & $80.89(1)$ & $99.1(3)_{-5 \%}^{+4 \%}$ \\
14 & $88.76(2)$ & $108.1(3)_{-5 \%}^{+3 \%}$ \\
\hline
\end{tabular}

Table 1. LO and NLO cross sections for diphoton production at the LHC with the acceptance cuts of eq. ( 4.3), as a function of $\sqrt{s}$. The Monte Carlo integration error on each prediction is shown in parentheses. For the NLO results the theoretical scale uncertainty is computed according to the procedure described in the text and is shown as a percentage deviation.

\subsection{Results}

As a point of reference, we first consider the cross section for unisolated photons at the LHC, for various centre-of-mass energies. We apply only basic acceptance cuts on the two photons,

$$
p_{T}^{\gamma}>25 \mathrm{GeV}, \quad\left|\eta_{\gamma}\right|<5
$$

The cross sections we report are completely inclusive in any additional parton radiation. For our theoretical predictions we choose renormalisation $\left(\mu_{R}\right)$, factorisation $\left(\mu_{F}\right)$ and fragmentation scales $\left(M_{F}\right)$ all equal to the diphoton invariant mass, $m_{\gamma \gamma}$. The results of our study at LO and NLO are shown in table 1, where the percentage uncertainties quoted on the NLO cross sections are estimated by varying all scales simultaneously by a factor of two in each direction. The inclusion of both $g g$ and $q \bar{q}$ processes in the LO result, and the next order corrections to both at NLO, results in only a mild 20-30\% increase in the cross section at NLO. Moreover, these predictions are rather stable with respect to scale variations over the range studied, with deviations in each direction of at most $6 \%$.

We now wish to investigate a more realistic set of cuts in which the photon is isolated. Since this final state is particularly interesting in the context of a low-mass Higgs search [25], for illustration we adopt the set of cuts used in an early search by the ATLAS collaboration [30]. The photons are required to be relatively central and subject to staggered transverse momentum cuts,

$$
p_{T}^{\gamma_{1}}>40 \mathrm{GeV}, \quad p_{T}^{\gamma_{2}}>25 \mathrm{GeV}, \quad\left|\eta_{\gamma_{i}}\right|<2.5,
$$




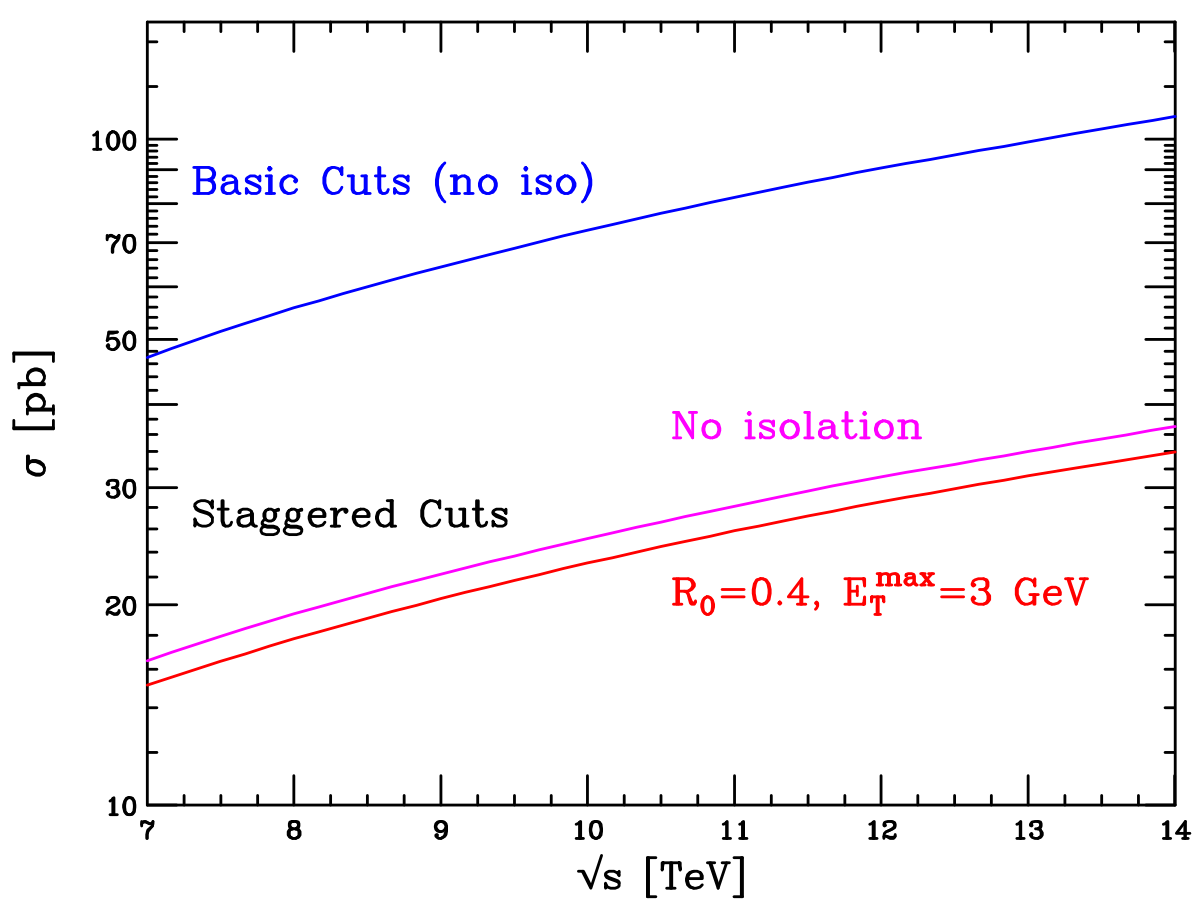

Figure 2. The NLO prediction for the diphoton cross section (in picobarns) as a function of the centre of mass energy, $\sqrt{s}$. The cross sections are shown for three sets of cuts: only the basic cuts of eq. ( 4.3) (upper, blue curve); the staggered cuts of eq. (4.4) (middle, magenta curve); the isolated photon cross section, eqs. ( $4.4,4.5)$ (lower, red curve).

and are isolated using a fixed maximum hadronic energy in a photon cone (c.f. eq. (2.1)),

$$
R_{0}=0.4, \quad E_{T}^{\max }=3 \mathrm{GeV} .
$$

The effect of these cuts, as a function of $\sqrt{s}$, is shown in figure 2 . The effect of the staggered cuts, eq. (4.4), is to lower the cross section by approximately a factor of three compared to the basic cuts of eq. (4.3). The isolation condition, eq. (4.5), further reduces the cross section from the nominal unisolated prediction by about $9 \%$. We note that this reduction is smaller than one would typically expect when going from unisolated to isolated cross sections. This is due mostly to the staggered cuts which favour the 3 particle final state.

In fact the cross section is rather insensitive to the amount of transverse hadronic energy allowed in the isolation cone. This is illustrated in figure 3, which shows the dependence of the cross section on the value of the isolation parameter $E_{T}^{\max }$. As a result of the small variation over this range, isolation cuts of the form $E+\delta p_{T}^{\gamma}$ where $E$ and $\delta$ are constants and $\delta \ll E$ are well-approximated theoretically by using a simple constant $E_{T}^{\max }=E+\delta p_{T, \min }^{\gamma}$.

For the cross sections presented so far we have chosen to set all scales entering our calculation equal to the invariant mass of the two photons, $\mu_{R}=\mu_{F}=M_{F} \equiv \mu_{0}$, with $\mu_{0}=$ $m_{\gamma \gamma}$. In order to illustrate the impact of this choice, in figure 4 we show the dependence of the theoretical predictions on the common scale $\mu$ when it is varied by a factor of four about $\mu_{0}$. In addition to the scale dependence of the total predictions we also consider the 


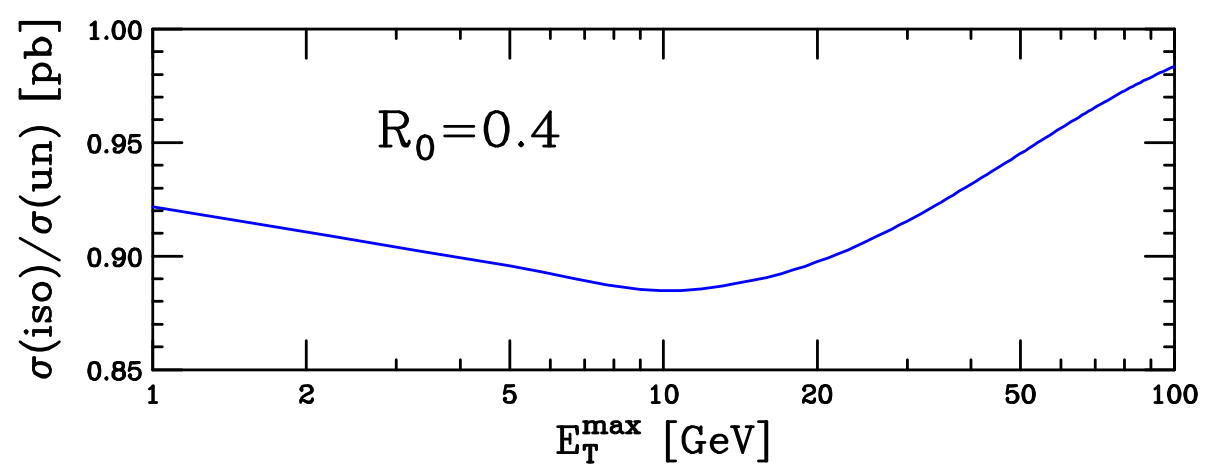

Figure 3. The fraction of the unisolated diphoton cross section that remains when the photon is isolated, as a function of the maximum amount of transverse hadronic energy allowed in the photon isolation cone, $E_{T}^{\max }$. The centre of mass energy is $\sqrt{s}=7 \mathrm{TeV}$ and photons are identified according to the staggered cuts of eq. (4.4). The radius of the isolation cone is $R_{0}=0.4$.

variation with $\mu$ of the individual partonic channels that appear at each order. Although the scale dependence of the individual partonic processes is typically quite large (for example, for the $q \bar{q}$ and $q g$ initiated processes at NLO), the sum over all contributions is relatively scale-independent. The LO cross section in particular has a tiny variation in this range. The fact that the NLO corrections are large and not reproducible for any choice of scale considered at LO serves as a reminder that the scale variation is not indicative of the theoretical uncertainty at that order. We also note the large K-factor when going from LO to NLO $(\sim 3.2)$, which is in stark contrast to the mild corrections observed when imposing only basic acceptance cuts (c.f. table 1). This difference can easily be understood from the nature of the cuts in eqs. $(4.4,4.5)$. For the Born and virtual contributions the staggered $p_{T}$ cut is effectively a $p_{T}^{\gamma_{2}}>40 \mathrm{GeV}$ cut due to the $2 \rightarrow 2$ kinematics. Photons of $p_{T}<40 \mathrm{GeV}$ can only be produced by fragmentation or real radiation diagrams in which a parton is available to balance the staggered transverse momenta. As a result these cuts strongly favour real radiation diagrams, a fact that is also evident from the size of the $q g$ contribution in figure 4 . We can reduce the $K$-factor and therefore increase the reliability of the calculation by choosing minimum $p_{T}$ cuts for the photons that are less staggered. As we reduce the difference $\Delta$ between the two photon energy cuts in eq. (4.4), finite terms of the form $\Delta \log \Delta$ are generated [31]. In the small $\Delta$ region these terms, when present, would require resummation. Setting the cut for one of the photons at $40 \mathrm{GeV}$, we find that the $\Delta \log \Delta$ terms in the total cross section are insignificant for $|\Delta|>3 \mathrm{GeV}$. Our overall recommendation therefore is to choose staggered cuts that are of the order of a few $\mathrm{GeV}$. The distribution of the diphoton invariant mass is free of $\Delta \log \Delta$ terms in the Higgs search region far above threshold, so for this case equal $p_{T}$ cuts for the photons are acceptable.

Finally, we consider predictions for the diphoton invariant mass distribution, a key ingredient in the search for a light Higgs boson. Our results for $\sqrt{s}=7 \mathrm{TeV}$ and the cuts of eqs. $(4.4,4.5)$ are shown in figure 5 . It is clear that, in order to provide a good prediction for this distribution, one must include not only the gluon-gluon initiated process but also the NLO corrections throughout. 

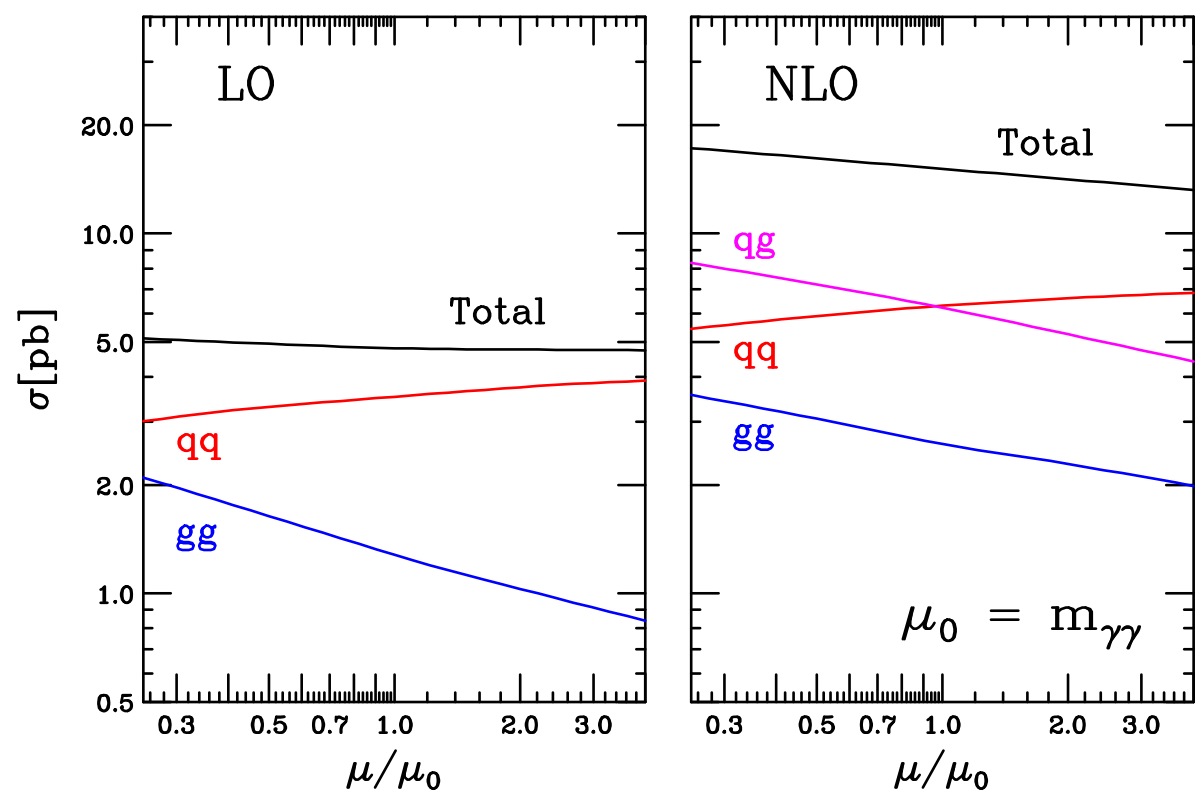

Figure 4. Dependence of the LO and NLO diphoton cross sections at $\sqrt{s}=7 \mathrm{TeV}$ (in pb) on the scale choice $\mu$. We vary $\mu \equiv \mu_{R}=\mu_{F}=M_{F}$ about the central scale choice $\mu_{0}=m_{\gamma \gamma}$. Total cross sections are shown in black whilst colours are used to denote the scale dependence of particular initial states: quark-antiquark (red), quark-gluon (magenta), gluon-gluon (blue). Photons are defined and isolated according to eqs. (4.4, 4.5).

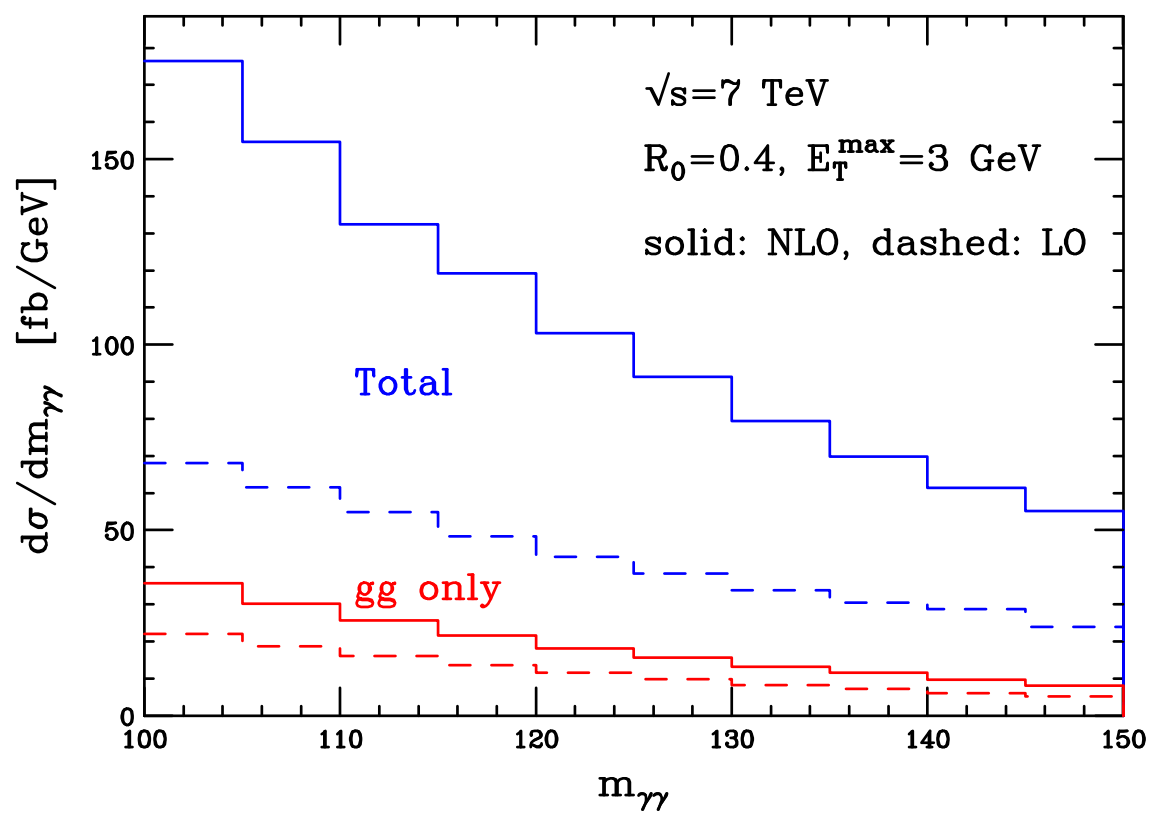

Figure 5. The diphoton invariant mass distribution at $\sqrt{s}=7 \mathrm{TeV}$ (in $\mathrm{fb} / \mathrm{GeV}$ ). We apply the staggered cuts described in the text and indicate LO results with dashed curves and NLO results with solid curves. The two upper (blue) curves show the full predictions at a given order, while the lower (red) curves indicate the gluon-gluon initiated contributions only. 


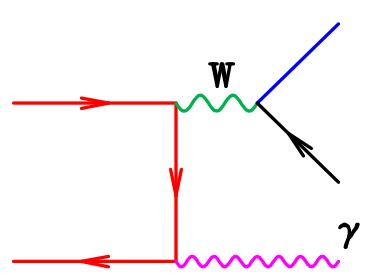

(a)

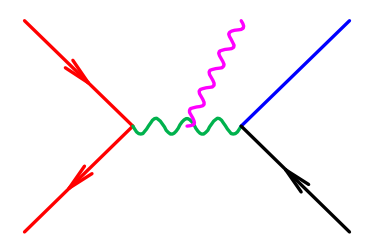

(c)

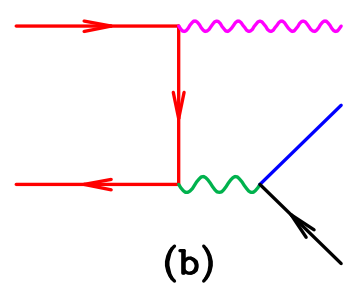

(b)

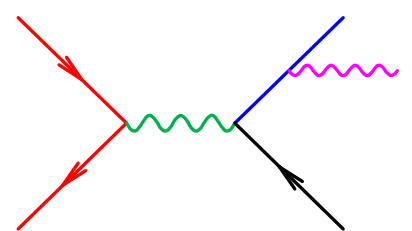

(d)

Figure 6. Leading order diagrams for $W(\rightarrow \ell \nu) \gamma$ production. The diagrams (a), (b) and (c) can be considered as radiation in the production process, while the final diagram (d) corresponds to photon radiation from the lepton in the $W$ decay.

\section{$5 \quad W^{ \pm} \gamma$ production}

\subsection{Description of the calculation}

The production of a $W$ boson and a photon proceeds at Born level via quark-antiquark annihilation,

$$
q+\bar{q}^{\prime} \rightarrow W^{ \pm} \gamma
$$

This process was first calculated several decades ago [32], with the effect of radiative corrections subsequently accounted for in ref. [33]. Since then the subject has been revisited several times. A fully differential Monte Carlo implementation of the NLO result is presented in ref. [34], making use of the helicity amplitudes calculated in ref. [35]. Spin correlations in the decay of the $W$ boson are included although no photon radiation from the lepton is allowed. Electroweak corrections to this process [36] and NLO QCD corrections to the related $W \gamma+$ jet final state have also been computed [37].

In this section we present results using the current implementation of this process in MCFM. The diagrams that contribute to this process at leading order are shown in figure 6 . The next-to-leading order diagrams are obtained by dressing these diagrams with both virtual and real gluon radiation. The contribution to the full amplitude arising from three of these diagrams is readily obtained from the helicity amplitudes of ref. [35]. The final diagram, including appropriate dressings that are straightforward to compute, accounts for the additional contribution from photon radiation in the leptonic decay of the $W$ boson. The resulting amplitude retains full spin correlations in the decay. 


\begin{tabular}{|c|c|c|c|c|}
\hline$\sqrt{s}[\mathrm{TeV}]$ & $\sigma^{L O}\left(e^{+} \nu \gamma\right)[\mathrm{pb}]$ & $\sigma^{N L O}\left(e^{+} \nu \gamma\right)[\mathrm{pb}]$ & $\sigma^{L O}\left(e^{-} \bar{\nu} \gamma\right)[\mathrm{pb}]$ & $\sigma^{N L O}\left(e^{-} \bar{\nu} \gamma\right)[\mathrm{pb}]$ \\
\hline 7 & $23.02(6)$ & $30.1(1)_{-6 \%}^{+5 \%}$ & $15.46(5)$ & $21.1(1)_{-8 \%}^{+4 \%}$ \\
8 & $26.86(8)$ & $35.1(2)_{-7 \%}^{+3 \%}$ & $18.53(7)$ & $24.6(1)_{-6 \%}^{+5 \%}$ \\
9 & $30.62(8)$ & $39.6(2)_{-7 \%}^{+5 \%}$ & $21.26(8)$ & $28.4(2)_{-6 \%}^{+4 \%}$ \\
10 & $34.6(1)$ & $44.2(4)_{-6 \%}^{+5 \%}$ & $24.13(8)$ & $32.2(2)_{-8 \%}^{+3 \%}$ \\
11 & $38.4(1)$ & $48.8(3)_{-8 \%}^{+4 \%}$ & $27.1(1)$ & $35.7(2)_{-6 \%}^{+4 \%}$ \\
12 & $42.2(1)$ & $54.0(4)_{-8 \%}^{+3 \%}$ & $30.2(1)$ & $39.4(2)_{-6 \%}^{+5 \%}$ \\
13 & $45.9(1)$ & $57.7(4)_{-6 \%}^{+3 \%}$ & $33.1(1)$ & $43.6(3)_{-8 \%}^{+4 \%}$ \\
14 & $49.8(1)$ & $62.8(4)_{-9 \%}^{+5 \%}$ & $36.0(1)$ & $47.4(3)_{-8 \%}^{+4 \%}$ \\
\hline
\end{tabular}

Table 2. Cross sections for $W(\rightarrow \ell \nu) \gamma$ production as a function of energy, using only the cuts of eq. (5.5). The cross sections are calculated including the effects of photon radiation in the $W$ decay and the central values are obtained using $\mu_{R}=\mu_{F}=M_{F}=M_{W}$. The uncertainty is derived from the scale dependence, as described in the text.

\section{$5.2 \quad$ Results}

In order to define the final state for this process we apply a basic set of kinematic cuts,

$$
p_{T}^{\gamma}>10 \mathrm{GeV}, \quad R_{\ell \gamma}>0.7,
$$

and demand that the photon be isolated as before, $R_{0}=0.4$ and $E_{T}^{\max }=3$. In this subsection we consider $W$ bosons which decay leptonically. We do not apply any cuts to the leptons, except for the photon-lepton separation cut which ensures that the photonlepton collinear singularity is avoided. The resulting cross sections are given, as a function of $\sqrt{s}$, in table 2. We present results for the LO and NLO cross sections for $e^{+} \nu \gamma$ and $e^{-} \bar{\nu} \gamma$ separately. The cross sections have been calculated using a central scale choice of $\mu_{R}=\mu_{F}=M_{F}=M_{W}$, with upper and lower extrema obtained by evaluating the cross section at $\left\{\mu_{R}=M_{W} / 2, \mu_{F}=2 M_{W}\right\}$ and $\left\{\mu_{R}=2 M_{W}, \mu_{F}=M_{W} / 2\right\}$ respectively. The fragmentation scale is kept fixed at $M_{W}$ throughout since its variation does not lead to a significant change in our results over the range of interest. From this table we can readily extract our NLO prediction for the $W \gamma$ cross section (summed over both $W^{+}$and $W^{-}$) at current LHC operating energies with the cuts and isolation described above,

$$
\sigma^{N L O}(p p \rightarrow W \gamma+X) \times B R(W \rightarrow \ell \nu)=51.2_{-3.5}^{+2.3} \mathrm{pb} .
$$

This is to be compared with a recently-reported cross section from the CMS collaboration [3]. They find,

$$
\sigma^{C M S}(p p \rightarrow W \gamma+X) \times B R(W \rightarrow \ell \nu)=55.9 \pm 5.0 \text { (stat) } \pm 5.0 \text { (sys) } \pm 6.1 \text { (lumi) pb }
$$

in good agreement with the Standard Model expectation. 


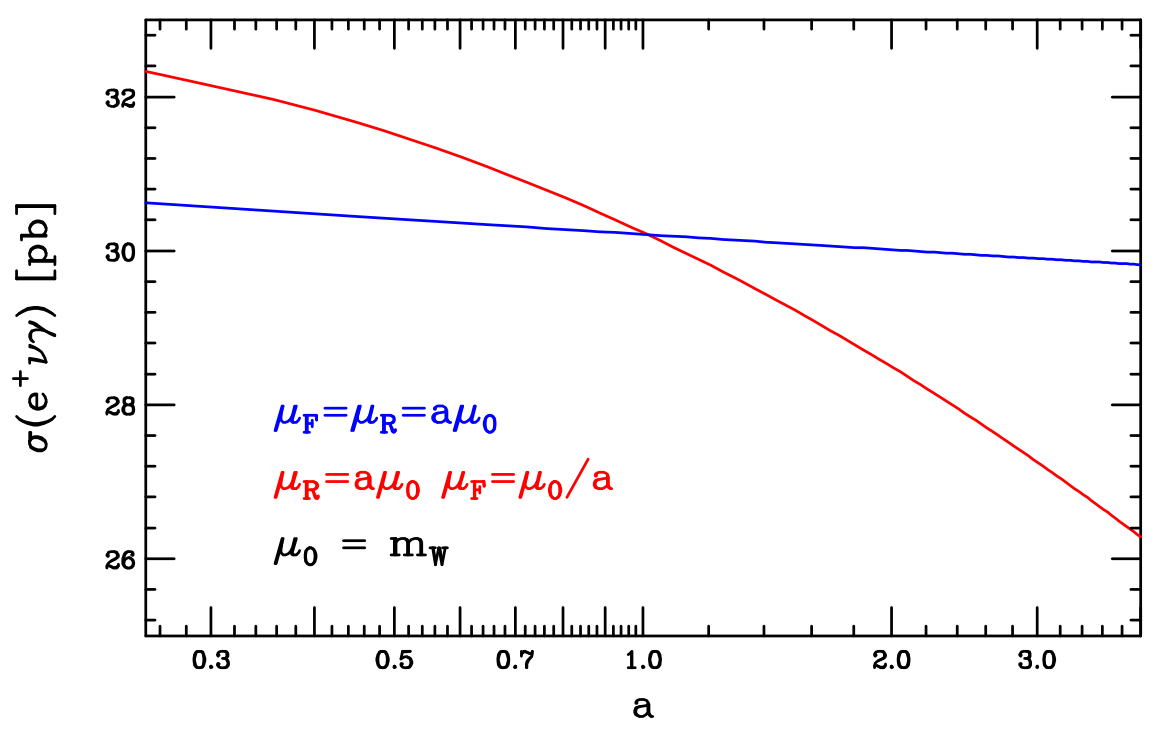

Figure 7. Scale variation for $W^{+}\left(\rightarrow e^{+} \nu\right) \gamma$ production, applying only the basic cuts of eq. (5.5). For the red curve we vary the factorisation and renormalisation functions in opposite directions, whilst for the blue curve we vary them in the same direction. The fragmentation scale is kept fixed at $M_{W}$.

Varying the factorisation and renormalisation scales in the manner that we have chosen requires some further justification since the normal theoretical preference is to change them together in the same direction. A comparison of these two choices, varying $\mu_{F}$ and $\mu_{R}$ in the same and opposite directions, is shown in figure 7 . We observe that there is essentially no change in the NLO $e^{+} \nu \gamma$ cross section when the scales are varied in the same direction, which is due to the qualitatively different behaviour of the contributing partonic states. The $q \bar{q}$ initial state is dominated by variations in the factorisation scale and grows with increasing $\mu_{F}$. Conversely, the $g q$ initial state depends most strongly on the renormalisation scale and decreases with increasing $\mu_{R}$. The combination of these two initial states results in a very small net scale dependence. Since this is simply a fortuitous cancellation and higher order corrections to the NLO cross section will likely not be bracketed by this small scale variation, we choose to vary the scales in opposite directions instead. We believe that this results in a more credible estimate of the theoretical uncertainty of the calculation.

At LHC centre of mass energies the dominant contributions to the cross sections that we have presented so far result from the radiation of a photon from the lepton in the $W$ decay. For studies of anomalous couplings of vector bosons to photons, and for the observation of radiation zeros in rapidity distributions, it is most useful to suppress this contribution. This is achieved by applying a cut on the transverse mass $\left(M_{T}\right)$ of the photon-lepton-MET system, $M_{T}>90 \mathrm{GeV}$. To investigate the role of lepton cuts on the cross section and distributions we will first present results for the cross section at $\sqrt{s}=7 \mathrm{TeV}$ including the 


\begin{tabular}{|c|c|c|c|c|c|}
\hline Decay & Cuts & $\sigma^{L O}\left(e^{+} \nu \gamma\right)$ & $\sigma^{N L O}\left(e^{+} \nu \gamma\right)$ & $\sigma^{L O}\left(e^{-} \bar{\nu} \gamma\right)$ & $\sigma^{N L O}\left(e^{-} \bar{\nu} \gamma\right)$ \\
\hline No FSR & Basic $\gamma$ & 4.88 & 8.74 & 3.15 & 6.01 \\
& $M_{T}$ cut & 1.99 & 3.78 & 1.26 & 2.66 \\
& Lepton cuts & 1.49 & 2.73 & 0.86 & 1.77 \\
\hline Full & Basic $\gamma$ & 23.0 & 30.1 & 15.5 & 21.1 \\
& $M_{T}$ cut & 2.12 & 3.94 & 1.34 & 2.75 \\
& Lepton cuts & 1.58 & 2.85 & 0.91 & 1.81 \\
\hline
\end{tabular}

Table 3. $W(\rightarrow \ell \nu) \gamma$ cross sections in picobarns at $\sqrt{s}=7 \mathrm{TeV}$ for the various scenarios described in detail in the text. Results in the upper half ("No FSR") correspond to neglecting diagrams containing photon radiation in the $W$ decay, while the cross sections in the lower half ("Full") include this effect. The cuts on the final state are specified in eqs. (5.5)-(5.7). Statistical errors are \pm 1 in the final digit.

$W$ decay for various sets of cuts. The three sets of cuts that we will consider are,

$$
\begin{aligned}
& \text { Basic Photon }: p_{T}^{\gamma}>10 \mathrm{GeV},\left|\eta_{\gamma}\right|<5, R_{\ell \gamma}>0.7, R_{0}=0.4, E_{T}^{\max }=3 \mathrm{GeV} . \\
& M_{T} \text { cut }: \text { Basic Photon }+M_{T}>90 \mathrm{GeV} . \\
& \text { Lepton cuts : } M_{T} \text { cut }+E_{T}^{\text {miss }}>25 \mathrm{GeV}, p_{T}^{\ell}>20 \mathrm{GeV},\left|\eta_{\ell}\right|<2.5
\end{aligned}
$$

For each of these sets of cuts we will perform our NLO calculation in two different ways. In the first case (no final-state radiation, "No FSR") we will omit diagram figure 6(d) corresponding to photon radiation in the $W$ decay (and its appropriate NLO dressings). Such an approach is natural if one demands that the lepton-neutrino system is produced exactly on the $W$ mass-shell. This corresponds to the approach taken in ref. [34]. This constraint cannot be implemented physically. For the second case ("Full") we follow our usual procedure and include this diagram and NLO counterparts. The results are summarised in table 3.

We observe that with just the basic cuts of eq. (5.5) the difference in predicted cross section between the two calculations is very large. At NLO the full result is over three times larger than the "No FSR" equivalent. As claimed earlier, applying the $M_{T}$ cut of eq. (5.6) significantly reduces this difference. The NLO cross section including radiation in the decay is about $3 \%$ higher. The quantity that is most relevant experimentally corresponds to the full cuts given in eq. (5.7). In that case the two calculations differ by at most $4 \%$ at NLO.

Including the final state radiation of photons not only significantly increases $W \gamma$ cross sections at the LHC, it also changes the character of the radiation zero [38] that is present in the amplitude. The signature of the radiation amplitude zero can be seen in the distribution of the pseudorapidity difference between the charged lepton and the photon. Our predictions for this distribution, using $\mu_{R}=\mu_{F}=M_{F}=M_{W}$ and applying the full lepton cuts of eq. (5.7), are shown in figure 8. The dashed blue curve in figure 8 represents the NLO rapidity difference with lepton cuts (eq. (5.7)), but with no cut on $M_{T}$ applied. We observe that the characteristic dip associated with the radiation zero has been completely 


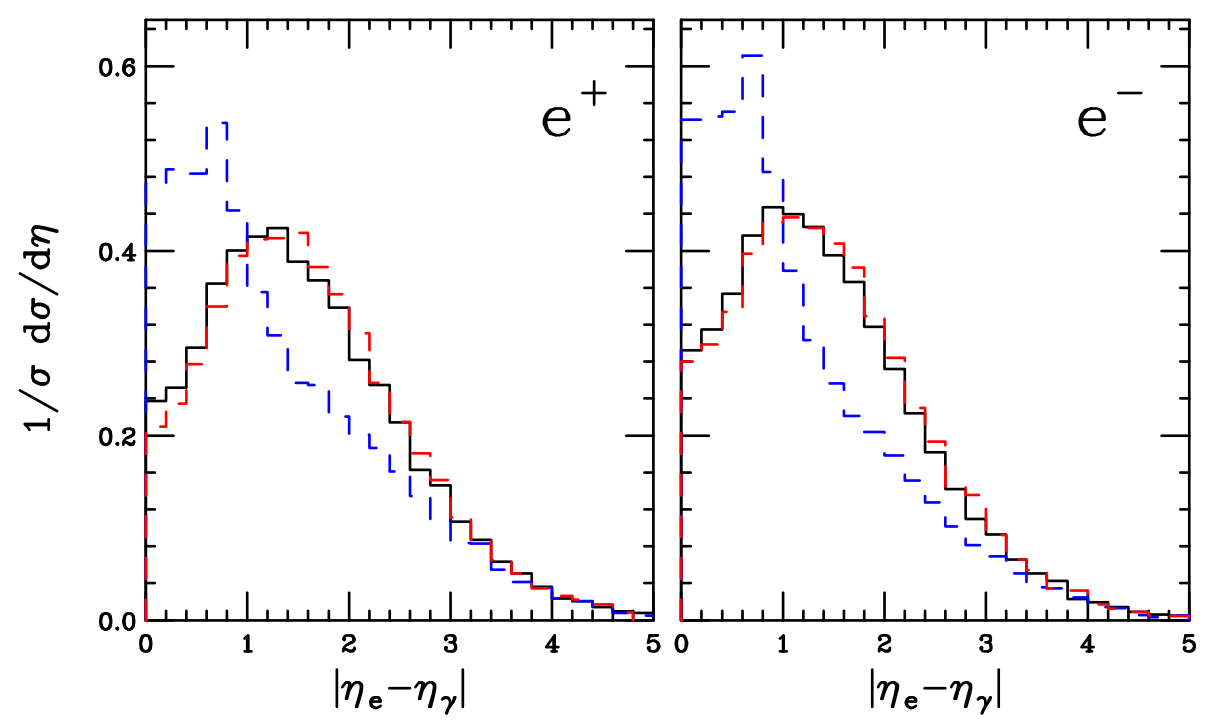

Figure 8. NLO Predictions for the pseudorapidity difference between the charged lepton and the photon in $W(\rightarrow \ell \nu) \gamma$ events, for three different levels of the calculation. For all curves we apply the lepton cuts of eq. ( 5.7). The black curve represents the complete NLO prediction. The red dashed curve represents the NLO prediction in the case where no photon radiation is allowed from the lepton ("No FSR"). The blue dashed curve has no cut on $M_{T}$, but keeps the cuts on the leptons.

filled in by the radiation of photons from the charged lepton. This is due to the fact that this configuration favours a collinear electron-photon pair so the rapidity difference between the two is usually small. Applying the $M_{T}$ cut (black curve) removes the majority of these configurations and the dip is restored. With the $M_{T}$ cut the NLO prediction from the full theory is similar to the result from the "No FSR" calculation (red curve).

\section{$6 \quad Z \gamma$ production}

\subsection{Description of the calculation}

The production of a $Z$ boson and a photon primarily occurs through the Born process,

$$
q+\bar{q} \rightarrow Z \gamma
$$

The next-to-leading order corrections to this were computed in refs. [33, 39] and later extended to the case of a decaying $Z$ boson in ref. [34]. Electroweak corrections to this process have also been computed [36, 40].

A further contribution arises from the process,

$$
g+g \rightarrow Z \gamma
$$

which proceeds via a quark loop. Since this contribution is finite it can be computed separately, as first detailed in refs. $[15,16]$. More recently this process has been computed including the leptonic decay of the $Z$ boson and other higher order contributions [17]. 
The results presented in this section are obtained using our current implementation in MCFM which is as follows. Strong corrections to the $q \bar{q}$ initiated process are fully included, also allowing additional contributions from fragmentation processes. The $g g$ process is included for five flavours of massless quarks. The contribution from massive top quark loops is suppressed by $1 / m_{t}^{4}$ and is therefore neglected. We find agreement with the large top-mass limit of the results presented in ref. [16], where the full dependence on the top and bottom quark masses has been kept.

Analytic expressions for the $g g \rightarrow Z \gamma$ amplitudes that we include may be obtained from existing results for $e^{+} e^{-} \rightarrow 4$ partons [27], as described in appendix B.

\subsection{Results}

We begin by assessing the impact of radiation in the decay of the $Z$ boson to charged leptons. As before we consider three sets of cuts to illustrate the difference between the two calculations. These are:

$$
\begin{aligned}
\text { Basic Photon : } & m_{e^{+} e^{-}}>50 \mathrm{GeV}, p_{T}^{\gamma}>10 \mathrm{GeV},\left|\eta_{\gamma}\right|<5, R_{\ell \gamma}>0.7, \\
& R_{0}=0.4, E_{T}^{\max }=3 \mathrm{GeV} . \\
M_{\ell \ell \gamma} \text { cut : } & \text { Basic Photon }+M_{\ell \ell \gamma}>100 \mathrm{GeV} . \\
\text { Lepton cuts : } & M_{\ell \ell \gamma} \text { cut }+p_{T}^{\ell}>20 \mathrm{GeV},\left|\eta_{\ell}\right|<2.5 .
\end{aligned}
$$

The first set of cuts, eq. (6.3), is very similar to the basic cuts for the $W \gamma$ process (eq. (5.5)) but with an additional dilepton invariant mass cut in order to select real $Z$ events. The cut on the transverse mass $M_{T}$ has been replaced with a cut on the invariant mass of the photon+leptons system. This reflects the fact that for $Z\left(\rightarrow \ell^{+} \ell^{-}\right) \gamma$ production all of the final state is reconstructed. Also the value of this mass cut must be slightly higher than the equivalent for $W \gamma$ (eq. (5.6)), to reflect the $\sim 10 \mathrm{GeV}$ higher mass of the $Z$ boson. The lepton cuts are identical, without of course any requirement on the missing transverse energy. These cuts are motivated by an early CMS study [3]. Our results for $\sqrt{s}=7 \mathrm{TeV}$, shown in table 4 , indicate that the $M_{\ell \ell \gamma}$ cut is reasonably effective at removing the contribution to the cross section from photons in the $Z$ decay. In the presence of the full lepton cuts, given in eq. (6.5), including photon radiation in the decay increases the cross section by about $15 \%$ at NLO. This is a larger difference than for $W \gamma$ production, which is to be expected since radiation may occur from both decay products.

We now turn to the issue of the dependence of the cross section on the centre-of-mass energy $\sqrt{s}$ and the estimation of the theoretical uncertainty from scale variation. As is the case for the $W \gamma$ cross sections of the previous section, we find that varying all scales by a factor of two about the central value of $M_{Z}$ results in a very small scale dependence. This is a result of the same accidental cancellation between the scaling behaviours of the component partonic cross sections. The scale uncertainties are therefore obtained by keeping $M_{F}=M_{Z}$ (since the fragmentation contribution is itself very small) and using $\left\{\mu_{R}=M_{Z} / 2, \mu_{F}=2 M_{Z}\right\}$ and $\left\{\mu_{R}=2 M_{Z}, \mu_{F}=M_{Z} / 2\right\}$ for the upper and lower extrema respectively. Our results are shown in table 5. Finally, we can once again compare our NLO prediction for the total $Z \gamma$ cross section to a measurement already made at the LHC. 


\begin{tabular}{|c|c|c|c|}
\hline Decay & Cuts & $\sigma^{L O}\left(e^{+} e^{-} \gamma\right)$ & $\sigma^{N L O}\left(e^{+} e^{-} \gamma\right)$ \\
\hline \multirow{2}{*}{ No FSR } & Basic $\gamma$ & $1.67(0)$ & $2.33(0)$ \\
& $M_{\ell \ell \gamma}$ cut & $1.67(0)$ & $2.29(0)$ \\
& Lepton cuts & $0.82(0)$ & $1.17(0)$ \\
\hline \multirow{2}{*}{ Full } & Basic $\gamma$ & 7.84 & 9.83 \\
& $M_{\ell \ell \gamma}$ cut & $2.08(0)$ & 2.81 \\
& Lepton cuts & $0.99(0)$ & $1.39(0)$ \\
\hline
\end{tabular}

Table 4. $Z\left(\rightarrow e^{+} e^{-}\right) \gamma$ cross sections in picobarns at $\sqrt{s}=7 \mathrm{TeV}$ for the various scenarios described in detail in the text. Results in the upper half ("No FSR") correspond to neglecting diagrams containing photon radiation in the $Z$ decay, while the cross sections in the lower half ("FSR") include this effect. The cuts on the final state are specified in eqs. (6.3)-(6.5). Statistical errors, unless otherwise indicated, are \pm 1 in the final digit.

\begin{tabular}{|c|c|c|}
\hline$\sqrt{s}[\mathrm{TeV}]$ & $\sigma^{L O}\left(e^{+} e^{-} \gamma\right)[\mathrm{pb}]$ & $\sigma^{N L O}\left(e^{+} e^{-} \gamma\right)[\mathrm{pb}]$ \\
\hline 7 & $7.84(1)$ & $9.83(1)_{-4.7 \%}^{+3.6 \%}$ \\
8 & $9.23(1)$ & $11.48(1)_{-5.1 \%}^{+3.5 \%}$ \\
9 & $10.65(2)$ & $13.10(1)_{-5.4 \%}^{+3.6 \%}$ \\
10 & $12.10(2)$ & $14.72(1)_{-5.7 \%}^{+3.7 \%}$ \\
11 & $13.56(2)$ & $16.38(2)_{-6.1 \%}^{+3.6 \%}$ \\
12 & $15.01(3)$ & $18.00(2)_{-6.2 \%}^{+3.5 \%}$ \\
13 & $16.50(3)$ & $19.61(2)_{-6.6 \%}^{+3.6 \%}$ \\
14 & $17.97(3)$ & $21.20(2)_{-6.6 \%}^{+3.7 \%}$ \\
\hline
\end{tabular}

Table 5. Cross sections for $Z\left(\rightarrow e^{+} e^{-}\right) \gamma$ production as a function of energy, using only the cuts of eq. (6.3). The cross sections are calculated including the effects of photon radiation in the $Z$ decay and the central values are obtained using $\mu_{R}=\mu_{F}=M_{F}=M_{Z}$. The uncertainty is derived from the scale dependence, as described in the text.

From table 5 we see that our NLO prediction for the cross section at $7 \mathrm{TeV}$ and using the cuts of eq. (6.3) is,

$$
\sigma^{N L O}(Z \gamma) \times B R\left(Z \rightarrow \ell^{-} \ell^{+}\right)=9.83_{-0.46}^{+0.35} \mathrm{pb}
$$

The corresponding result reported by the CMS collaboration is [3],

$$
\sigma^{C M S}(Z \gamma) \times B R\left(Z \rightarrow \ell^{-} \ell^{+}\right)=9.3 \pm 1.0(\text { stat }) \pm 0.6 \text { (syst) } \pm 1.0 \text { (lumi) pb }
$$




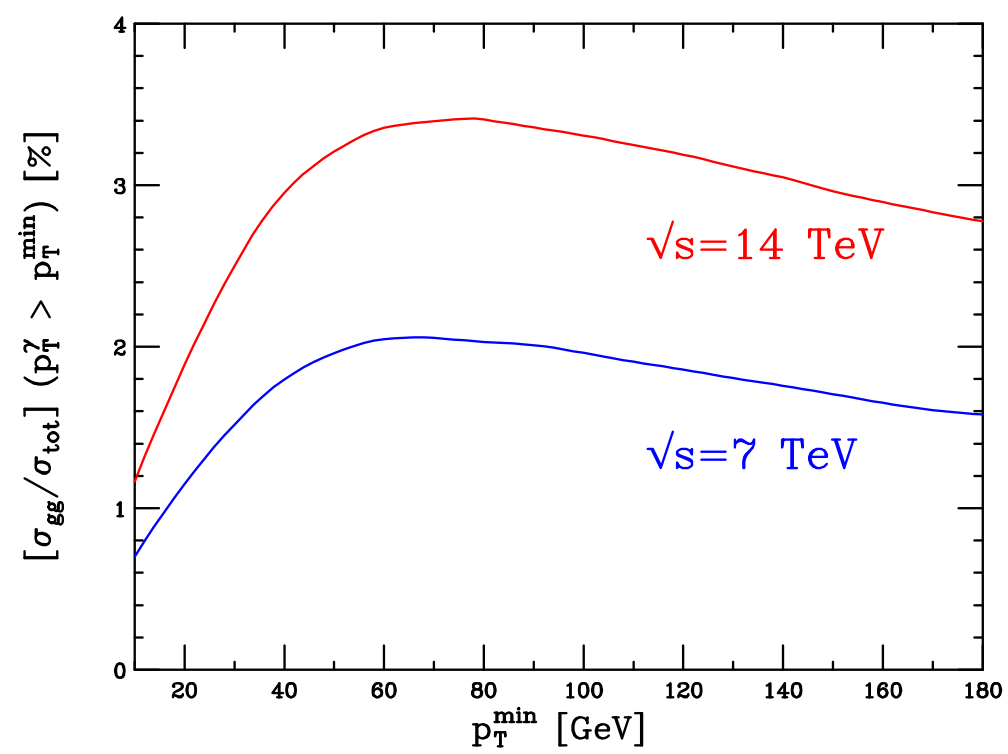

Figure 9. The percentage of the $Z\left(\rightarrow e^{+} e^{-}\right) \gamma$ cross section using the cuts of eq. ( 6.5) contributed by the gluon-gluon initiated diagrams, as a function of the minimum photon $p_{T}$ allowed in the events. The upper (red) curve is for $\sqrt{s}=14 \mathrm{TeV}$ while the lower (blue) curve corresponds to $\sqrt{s}=7 \mathrm{TeV}$.

which is already in good agreement within errors.

We conclude with an investigation of the importance of the gluon-gluon contribution in phenomenological studies. We shall use the full set of cuts given in eq. (6.5) as indicative of the appropriate experimental acceptance at the LHC. In that case we note that the relative effect of adding these diagrams is small since the cross section is dominated by regions of low $p_{T}^{\gamma}$ that are enhanced for the $q \bar{q}$ process but not for the loop-induced $g g$ diagrams. However, as one moves to moderate values of $p_{T}^{\gamma}$ one would expect the relative size of the gluon-gluon contribution to grow. This is exactly the behaviour that we observe in figure 9 , with the $g g$ fraction falling again at higher $p_{T}^{\gamma}$ due to the behaviour of the parton fluxes. We also see that, as expected, the gluon-gluon contribution is more important at $14 \mathrm{TeV}$, although it is still at most $3.5 \%$ of the total NLO cross section.

\section{$7 \quad W W$ production}

\subsection{Description of the calculation}

The production of a pair of $W$ bosons is an important channel, in part because of its role as a background to Higgs boson searches in which the Higgs decays into $W$ pairs. The total cross section for the process,

$$
q+\bar{q} \rightarrow W^{+} W^{-},
$$

was first calculated in the Born approximation in ref. [41], with strong corrections to it given in refs. [42-44]. These processes are included in MCFM at NLO using the one-loop 
amplitudes presented in ref. [35]. Phenomenological NLO results for the Tevatron and the LHC operating at $\sqrt{s}=14 \mathrm{TeV}$ have been presented in refs. [1, 45]. NLO results are also available for the processes $W^{+} W^{-}+$jet $[46,47], W^{+} W^{+}+2$ jets $[48]$ and $W^{+} W^{-}+$ 2 jets [49].

The contribution for the process,

$$
g+g \rightarrow W^{+} W^{-},
$$

was first calculated in refs. $[18,19]$. A more recent analysis of these contributions is given in ref. [21] where off-shell effects of the vector bosons and their subsequent decays are taken into account. Finally, the most complete analysis of these contributions to date is given in ref. [22] where the effect of massive quarks circulating in the loop is included. The authors find that the effect of including the third $(t, b)$ isodoublet increases the gluon-gluon contribution by at most a factor of $12 \%$ at the $14 \mathrm{TeV}$ LHC.

The results presented in this section are obtained using our current implementation in MCFM which is as follows. Strong corrections to the $q \bar{q}$ initiated process are fully included, with additional contributions from singly resonant diagrams as described in ref. [1]. Since the contribution from the $(t, b)$ isodoublet to the $g g$ initiated process is small - certainly much smaller than the residual uncertainty resulting from the $O\left(\alpha_{s}^{2}\right)$ nature of the contribution - the $g g$ process is included for two massless generations only. Our results for the $g g$ process are in complete agreement with the equivalent two generation results presented in ref. [22]. As can be seen from table 2 therein, the final cross section summed over $q \bar{q}$ and $g g$ channels is smaller than the three generation result by $0.5 \%$.

The inclusion of the $g g$ contribution with massless quarks in the loop is straightforward. The amplitudes can be obtained by simply recycling compact analytic expressions for certain contributions to the process $e^{+} e^{-} \rightarrow 4$ partons presented in ref. [27]. The precise relations are given in appendix B.

\subsection{Results}

We begin our discussion of $W W$ production by presenting the cross section as a function of $\sqrt{s}$ in table 6 . The values are obtained by evaluating the cross section with a central scale choice of $\mu_{R}=\mu_{F}=M_{W}$. Scale dependence is illustrated by presenting percentage deviations from the central value as the scales are changed simultaneously by a factor of two in each direction. The $W$ bosons are kept exactly on-shell and no decays are included for the cross sections presented in this table. We note that as for the other diboson cross sections the NLO corrections are typically large, enhancing the LO prediction by about a factor of 1.6. From the table, our NLO prediction for the total $W W$ cross section at $\sqrt{s}=7 \mathrm{TeV}$ is,

$$
\sigma^{N L O}=47.0_{-1.5}^{+2.0} \mathrm{pb} .
$$

Although the general-purpose detectors at the LHC have collected only a handful of such events, both ATLAS [4] and CMS [5] have already reported first measurements of this cross 


\begin{tabular}{|c|c|c|}
\hline$\sqrt{s}[\mathrm{TeV}]$ & $\sigma^{L O}\left(W^{+} W^{-}\right)[\mathrm{pb}]$ & $\sigma^{N L O}\left(W^{+} W^{-}\right)[\mathrm{pb}]$ \\
\hline 7 & $29.51(1)$ & $47.04(2)_{-3.2 \%}^{+4.3 \%}$ \\
8 & $35.56(1)$ & $57.25(2)_{-2.8 \%}^{+4.1 \%}$ \\
9 & $41.75(2)$ & $67.82(3)_{-2.8 \%}^{+3.8 \%}$ \\
10 & $48.07(2)$ & $78.70(3)_{-2.5 \%}^{+3.6 \%}$ \\
11 & $54.53(2)$ & $89.80(4)_{-2.5 \%}^{+3.3 \%}$ \\
12 & $61.10(3)$ & $101.14(5)_{-2.4 \%}^{+3.1 \%}$ \\
13 & $67.74(3)$ & $112.64(5)_{-2.3 \%}^{+3.0 \%}$ \\
14 & $74.48(4)$ & $124.31(6)_{-2.0 \%}^{+2.8 \%}$ \\
\hline
\end{tabular}

Table 6. Total cross sections for $W W$ production as a function of energy. Renormalisation and factorisation scales are set to $M_{W}$. Upper and lower limits are obtained by varying the scales by a factor of two in each direction. Vector bosons are kept on-shell, with no branching ratios applied

section. They find,

$$
\begin{aligned}
\sigma^{A T L A S}(W W) & =41_{-16}^{+20} \text { (stat) } \pm 5 \text { (syst) } \pm 1 \text { (lumi) pb } \\
\sigma^{C M S}(W W) & =41.1 \pm 15.3 \text { (stat) } \pm 5.8 \text { (syst) } \pm 4.5 \text { (lumi) pb }
\end{aligned}
$$

both of which are clearly compatible with the SM prediction.

A measurement of the $W W$ cross section at the LHC typically involves a jet veto to reduce the abundant top background $[4,5]$. Since a jet-veto can change the relative size of the NLO corrections we will study the dependence of the NLO cross section on the transverse momentum scale used to veto jets, $p_{T}^{\text {veto }}$. For our purposes here we define the jet veto as a veto on all jets with $p_{T}>p_{T}^{\text {veto }}$ that satisfy the rapidity requirement $\left|\eta_{j}\right|<5$. It is useful to consider the action of the jet veto under two sets of cuts,

$$
\begin{aligned}
\text { Basic } W W: & p_{T}^{\ell}>20 \mathrm{GeV},\left|\eta^{\ell}\right|<2.5, E_{T}^{\text {miss }}>20 \mathrm{GeV}, \\
\text { Higgs : } & \text { Basic } W W+m_{\ell \ell}<50 \mathrm{GeV}, \Delta \phi_{\ell \ell}<60^{\circ}, \\
& p_{T}^{\ell, \max }>30 \mathrm{GeV}, p_{T}^{\ell, \min }>25 \mathrm{GeV} .
\end{aligned}
$$

These cuts are typical of those used at the LHC to measure the total $W W$ cross section, (with the additional application of a jet-veto) and those used to search for a Higgs boson [5]. The precise nature of the Higgs search cuts are dependent on the putative mass of the Higgs boson so here we have selected a set used for $m_{H}=160 \mathrm{GeV}$, when the decay to $W W$ is largest.

The ratio of the NLO to LO cross sections, as a function of $p_{T}^{v e t o}$ and for the two sets of cuts above, is shown in the upper panels of figure 10. Since the $g g$ initiated contribution does not contain any final state partons it is unaffected by the jet-veto at this order. As 

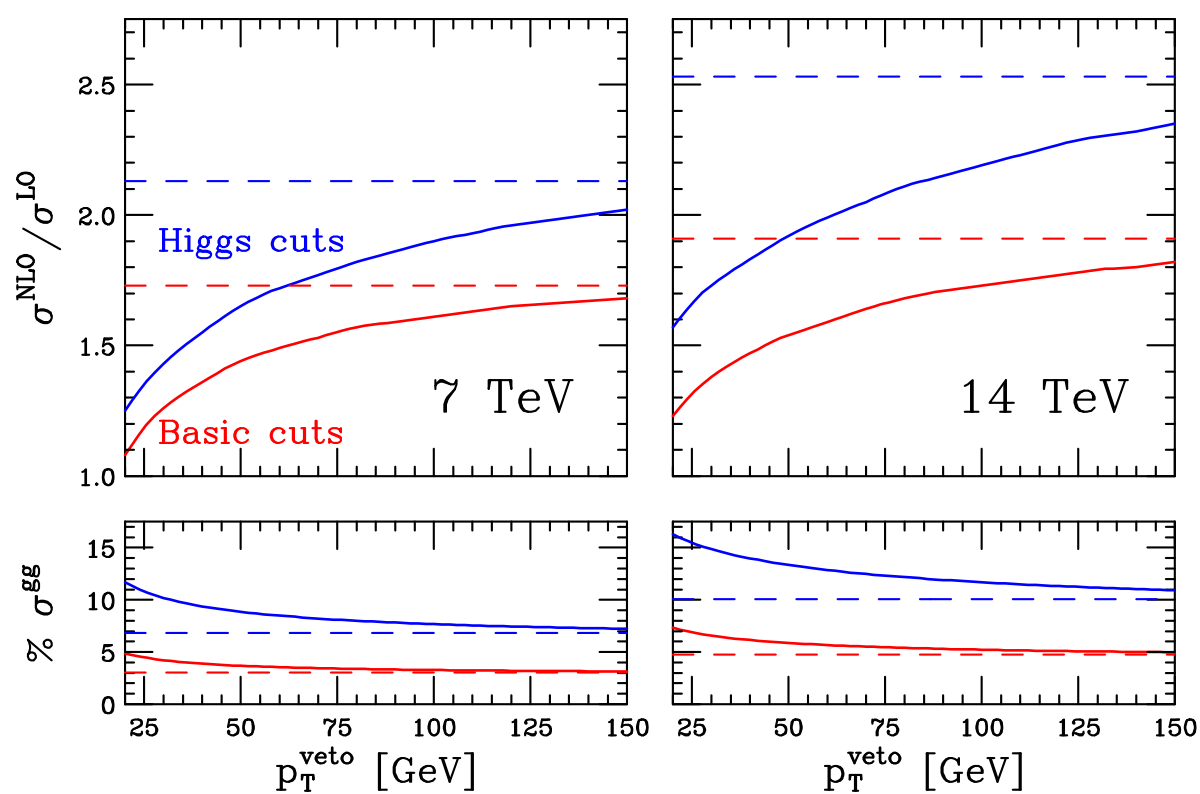

Figure 10. The ratio of NLO to LO (upper) and the percentage of the NLO cross section from the $g g$ initial state (lower) for $W W \rightarrow e^{+} \mu^{-} \nu_{e} \bar{\nu}_{\mu}$ production, as a function of the jet-veto $p_{T}^{v e t o}$. Results are shown using the basic cuts of eq. ( 7.6) (upper, blue curves) and the Higgs search cuts of eq. ( 7.7) (lower, red curves). The NLO to LO ratio and gluon percentage with no veto applied are shown as dashed lines on the plot. The dashed lines are thus the asymptotic values of the solid curves.

a result the relative importance of this contribution increases when a jet-veto is applied. We illustrate this by presenting the ratio $\sigma(g g) / \sigma^{N L O}$ in the lower panels of figure 10. As expected, the application of a jet-veto can reduce the $K$-factor considerably. For instance, applying a jet veto at $p_{T}^{\text {veto }}=20 \mathrm{GeV}$ reduces the inclusive $K$-factor by around $40 \%$. From figure 10 we also observe that the Higgs cuts increase both the impact of NLO corrections and the gluon initiated contributions. The importance of the gluon initiated terms for Higgs searches has been observed in previous studies [26]. Indeed these studies have shown that, at $\sqrt{s}=14 \mathrm{TeV}$ and with stricter cuts than those of eq. (7.7), the $g g$ contributions can be as large as $30 \%$ of the NLO cross section [26]. At $\sqrt{s}=7 \mathrm{TeV}$ and with cuts appropriate for this center of mass energy we find that the $g g$ contribution is around $12 \%$ of the total NLO cross section with a jet veto of $20 \mathrm{GeV}$, as shown in the lower panel of figure 10 . The values of the asymptotic limits of the jet veto curves shown in Fig 10, corresponding to the $K$-factor and $g g$ percentage with no veto applied, are collected in table 7 . For completeness we also include the corresponding predictions for the cross sections at LO and NLO.

\section{$8 \quad W^{ \pm} Z$ production}

\subsection{Description of the calculation}

The production of a $W Z$ pair proceeds at LO through the process,

$$
q+\bar{q}^{\prime} \rightarrow W^{ \pm} Z
$$




\begin{tabular}{|c|c|c|c|c|}
\hline$\sqrt{s}[\mathrm{TeV}]$ and cuts & $\sigma^{L O}\left(e^{+} \mu^{-} \nu_{e} \bar{\nu}_{\mu}\right)[\mathrm{fb}]$ & $\sigma^{N L O}\left(e^{+} \mu^{-} \nu_{e} \bar{\nu}_{\mu}\right)[\mathrm{fb}]$ & $K$-factor & $\%$ gg \\
\hline 7 (Basic) & 144 & 249 & 1.73 & 3.05 \\
7 (Higgs) & 7.14 & 15.19 & 2.13 & 6.85 \\
\hline 14 (Basic) & 296 & 566 & 1.91 & 4.73 \\
14 (Higgs) & 13.7 & 34.7 & 2.53 & 10.09 \\
\hline
\end{tabular}

Table 7. $W^{+}\left(\rightarrow e^{+} \nu_{e}\right) W^{-}\left(\rightarrow \mu^{-} \bar{\nu}_{\mu}\right)$ cross sections in femtobarns at LO and NLO, the resulting $K$-factor and the percentage of the NLO cross section originating from gluon initiated contributions. Results are shown for the Basic (eq. ( 7.6)) and Higgs (eq. ( 7.7)) cuts.

This process was first calculated to NLO in refs. [50, 51]. The inclusion of subsequent $W$ and $Z$ decays was added in ref. [44], partially including the effect of spin correlations. The full effect of spin correlations at NLO was later examined in refs. [1, 45], using the virtual amplitudes of ref. [35]. The QCD corrections to the process in which an additional jet is radiated are also now known [52].

The results presented in this section are obtained using the same implementation in MCFM as described in ref. [1]. In particular we include contributions from singly resonant diagrams that can be significant when one of the bosons is off-shell. The program includes both the contribution of a $Z$ and a virtual photon, when considering the decay to charged leptons. We note that charge conservation precludes any contribution from gluon-gluon diagrams of the type previously discussed for $W W$ production.

\subsection{Results}

The production of $W Z$ pairs provides a valuable test of the triple gauge boson couplings (for a recent example, see for instance ref. [53]) and is a source of SM background events, for example in SUSY trilepton searches $[54,55]$. It is also a background for SM Higgs searches in the case of leptonic decays, when one of the leptons is missed. In order to normalize the $W Z$ background to such searches, in table 8 we show results for the total cross section for $W Z$ production at the LHC, as a function of the centre of mass energy. Both renormalisation and factorisation scales are set to the mean vector boson mass, $\left(M_{W}+\right.$ $\left.M_{Z}\right) / 2$. Since the LHC is a proton-proton machine, the $W^{+} Z$ and $W^{-} Z$ cross sections are not equal, with the ratio $\sigma^{N L O}\left(W^{-} Z\right) / \sigma^{N L O}\left(W^{+} Z\right.$ ) varying between 0.56 (for $\sqrt{s}=$ $7 \mathrm{TeV}$ ) and 0.65 (at $14 \mathrm{TeV}$ ).

The study of boosted objects at the LHC has potential as an additional handle on searches for new physics, such as a Higgs boson [56] or supersymmetric particles [57]. A possible first step for such searches would be to validate the method by performing a similar analysis for known Standard Model particles. In this regard, $W Z$ production would be a natural proxy for associated Higgs production, $W H$, where the decay of the $Z$ boson to a bottom quark pair is a stand-in for the decay of a light Higgs boson. 


\begin{tabular}{|c|c|c|c|c|}
\hline$\sqrt{s}[\mathrm{TeV}]$ & $\sigma^{L O}\left(W^{+} Z\right)[\mathrm{pb}]$ & $\sigma^{N L O}\left(W^{+} Z\right)[\mathrm{pb}]$ & $\sigma^{L O}\left(W^{-} Z\right)[\mathrm{pb}]$ & $\sigma^{N L O}\left(W^{-} Z\right)[\mathrm{pb}]$ \\
\hline 7 & $6.93(0)$ & $11.88(1)_{-4.2 \%}^{+5.5 \%}$ & $3.77(0)$ & $6.69(0)_{-4.3 \%}^{+5.6 \%}$ \\
8 & $8.29(1)$ & $14.48(1)_{-4.0 \%}^{+5.2 \%}$ & $4.65(0)$ & $8.40(0)_{-4.1 \%}^{+5.4 \%}$ \\
9 & $9.69(1)$ & $17.18(1)_{-3.9 \%}^{+4.9 \%}$ & $5.57(0)$ & $10.21(0)_{-3.9 \%}^{+5.0 \%}$ \\
10 & $11.13(1)$ & $19.93(1)_{-3.7 \%}^{+4.8 \%}$ & $6.53(0)$ & $12.11(1)_{-3.7 \%}^{+4.8 \%}$ \\
11 & $12.56(1)$ & $22.75(2)_{-3.5 \%}^{+4.5 \%}$ & $7.51(0)$ & $14.07(1)_{-3.6 \%}^{+4.6 \%}$ \\
12 & $14.02(1)$ & $25.63(2)_{-3.3 \%}^{+4.3 \%}$ & $8.51(1)$ & $16.10(1)_{-3.4 \%}^{+4.4 \%}$ \\
13 & $15.51(2)$ & $28.55(2)_{-3.2 \%}^{+4.1 \%}$ & $9.53(1)$ & $18.19(1)_{-3.3 \%}^{+4.1 \%}$ \\
14 & $16.98(2)$ & $31.50(3)_{-3.0 \%}^{+3.9 \%}$ & $10.57(1)$ & $20.32(1)_{-3.1 \%}^{+3.9 \%}$ \\
\hline
\end{tabular}

Table 8. Total cross sections for $W Z$ production as a function of energy. Renormalisation and factorisation scales are set equal to the average mass of the $W$ and $Z$ i.e. $\mu_{R}=\mu_{F}=\left(M_{W}+M_{Z}\right) / 2$. Upper and lower percentage deviations are obtained by varying the scales around the central scale by a factor of two. The vector boson are kept on-shell, with no decays included.

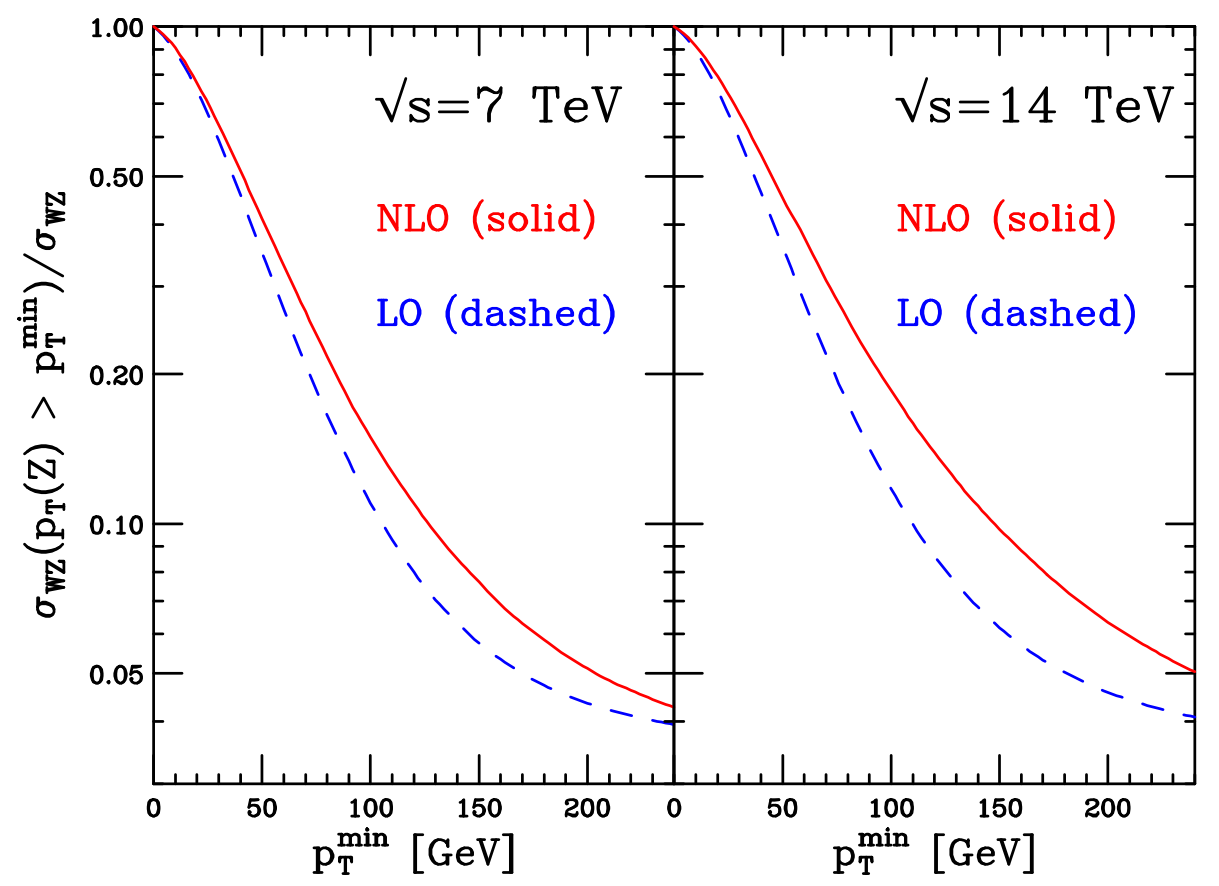

Figure 11. The fraction of the total $W Z$ cross section surviving a cut on the $Z$ boson transverse momentum, $p_{T}(Z)>p_{T}^{\min }$, at $\sqrt{s}=7 \mathrm{TeV}$ (left panel) and $\sqrt{s}=14 \mathrm{TeV}$ (right panel). The NLO prediction is shown as a solid red curve and the LO one is dashed blue.

To this end, in figure 11 we show the cross section for $W Z$ production as a function of the minimum $Z$ boson transverse momentum, at $\sqrt{s}=7$ and $\sqrt{s}=14 \mathrm{TeV}$. We observe 
that the number of boosted $Z$ bosons is sensitive both to the order in perturbation theory and the operating energy of the LHC. At both centre of mass energies the NLO prediction increases the number of high- $p_{T} Z$ bosons, although the effect is larger at $14 \mathrm{TeV}$. To emulate a typical boosted Higgs search, with a $p_{T}$ cut at $200 \mathrm{GeV}$, one thus retains about $5 \%$ of the total NLO cross section - similar to the fraction for a putative Higgs signal.

\section{$9 \quad Z Z$ production}

\subsection{Description of the calculation}

Although the production of $Z$ pairs is much smaller than the other diboson cross sections considered above, it still plays an important role as principal background to searches for a Higgs boson around the $Z$ pair threshold. The NLO corrections to the process,

$$
q+\bar{q} \rightarrow Z Z,
$$

were first calculated in refs. [58, 59], while the inclusion of spin correlations in the decays and phenomenology for the Tevatron and $14 \mathrm{TeV}$ LHC was presented in refs. [1, 45]. Contributions from a gluon-gluon initial state,

$$
g+g \rightarrow Z Z
$$

were first considered in refs. $[18,20]$. The inclusion of leptonic decays of the $\mathrm{Z}$ bosons was examined in refs. [23, 24] and later investigated in the context of Higgs boson searches [25, 26]. Furthermore, NLO results are also available for the closely-related $Z Z+$ jet process [60].

The results presented in this section are obtained using our current implementation in MCFM which is as follows. Strong corrections to the $q \bar{q}$ initiated process include singlyresonant contributions - a slight extension of the results presented in ref. [1] — and the $g g$ process is included for five massless flavours. The contribution from massive top quark loops is suppressed by $1 / m_{t}^{4}$ and is therefore neglected. This approximation results in gluon-gluon contributions that are $1 \%$ lower than those reported in refs. [25, 26], where the effects of massive top and bottom loops are included. ${ }^{2}$ Finally, we observe that all our amplitudes also contain contributions from virtual photons.

The basic amplitudes entering the calculation of the $g g$ contribution are simply related to those already discussed for the $g g \rightarrow W W$ process and are detailed in appendix B.

\subsection{Results}

We first present results for the dependence of the total cross section for $Z Z$ production as a function of $\sqrt{s}$. As was the case for similar studies in previous sections we keep the $Z$ bosons on-shell and do not include any decays. We choose a central scale choice of $\mu_{R}=\mu_{F}=M_{Z}$ and vary this central scale by a factor of two in each direction to obtain an estimate of the theoretical uncertainty. Our results are shown in table 9.

\footnotetext{
${ }^{2}$ We note that, when restricting our calculation to four massless flavours, our results are in complete agreement with the equivalent cross section quoted in ref. [25].
} 


\begin{tabular}{|c|c|c|}
\hline$\sqrt{s}[\mathrm{TeV}]$ & $\sigma^{L O}(Z Z)[\mathrm{pb}]$ & $\sigma^{N L O}(Z Z)[\mathrm{pb}]$ \\
\hline 7 & $4.17(0)$ & $6.46(0)_{-3.3 \%}^{+4.7 \%}$ \\
8 & $5.06(0)$ & $7.92(0)_{-3.0 \%}^{+4.7 \%}$ \\
9 & $5.98(0)$ & $9.46(0)_{-3.0 \%}^{+4.3 \%}$ \\
10 & $6.93(0)$ & $11.03(0)_{-2.9 \%}^{+4.1 \%}$ \\
11 & $7.90(0)$ & $12.65(1)_{-2.8 \%}^{+3.9 \%}$ \\
12 & $8.89(1)$ & $14.31(1)_{-2.7 \%}^{+3.6 \%}$ \\
13 & $9.89(1)$ & $15.99(1)_{-2.6 \%}^{+3.7 \%}$ \\
14 & $10.92(1)$ & $17.72(1)_{-2.5 \%}^{+3.5 \%}$ \\
\hline
\end{tabular}

Table 9. Total cross sections for $Z Z$ production as a function of energy. The renormalisation scale and factorisation scales are $\mu_{R}=\mu_{F}=M_{Z}$. Vector bosons are produced exactly on-shell and no decays are included.

The decay of a Higgs boson to two $Z$ 's, which subsequently decay to leptons, is a promising search channel at the LHC. This is due to the fact that the Higgs will decay to Z's (with a moderate branching ratio) over a large range of Higgs masses that are not presently excluded. In addition, the four lepton signature associated with $Z Z$ decay is experimentally clean. With Higgs searches in mind we apply the following cuts,

$$
p_{T}^{\ell_{1}, \ell_{2}}>20 \mathrm{GeV}, p_{T}^{\ell_{3}, \ell_{4}}>5 \mathrm{GeV},\left|\eta_{\ell}\right|<2.5, m_{\ell \ell}, m_{\ell^{\prime} \ell^{\prime}}>5 \mathrm{GeV} .
$$

In this definition of the cuts, $\ell_{1}$ and $\ell_{2}$ represent the two hardest leptons and $\ell_{3}$ and $\ell_{4}$ represent the two sub-leading leptons. The relevant distribution for the Higgs search is the invariant mass of the four-lepton system $\left(m_{4 \ell}\right)$, for which we present our predictions in figure 12. We show NLO predictions for both $\sqrt{s}=7 \mathrm{TeV}$ and $\sqrt{s}=14 \mathrm{TeV}$, as well as the contribution from the gluon-gluon diagrams alone.

From the figure we observe that, although the gluon initiated pieces are fairly important at the level of the total cross section, their effect in the region $m_{4 \ell}<2 M_{Z}$ is rather smaller (at the few percent level). As this threshold is crossed the percentage effect increases to around $7 \%(7 \mathrm{TeV})$ or $10 \%(14 \mathrm{TeV})$. Our results at $14 \mathrm{TeV}$ agree with the findings of a previous study in a similar kinematic range [26]. It is clear that the $g g$ initiated piece is most important as a background to Higgs bosons searches in the region $m_{H}>2 M_{Z}$.

\section{Conclusions}

In this paper we have provided NLO predictions for all diboson processes at the LHC, both at the current operating energy of $\sqrt{s}=7 \mathrm{TeV}$ and at higher energies appropriate for future running. The calculations are contained in the parton level code MCFM, which includes the implementation of $p p \rightarrow \gamma \gamma$ for the first time. In addition, where appropriate we have 


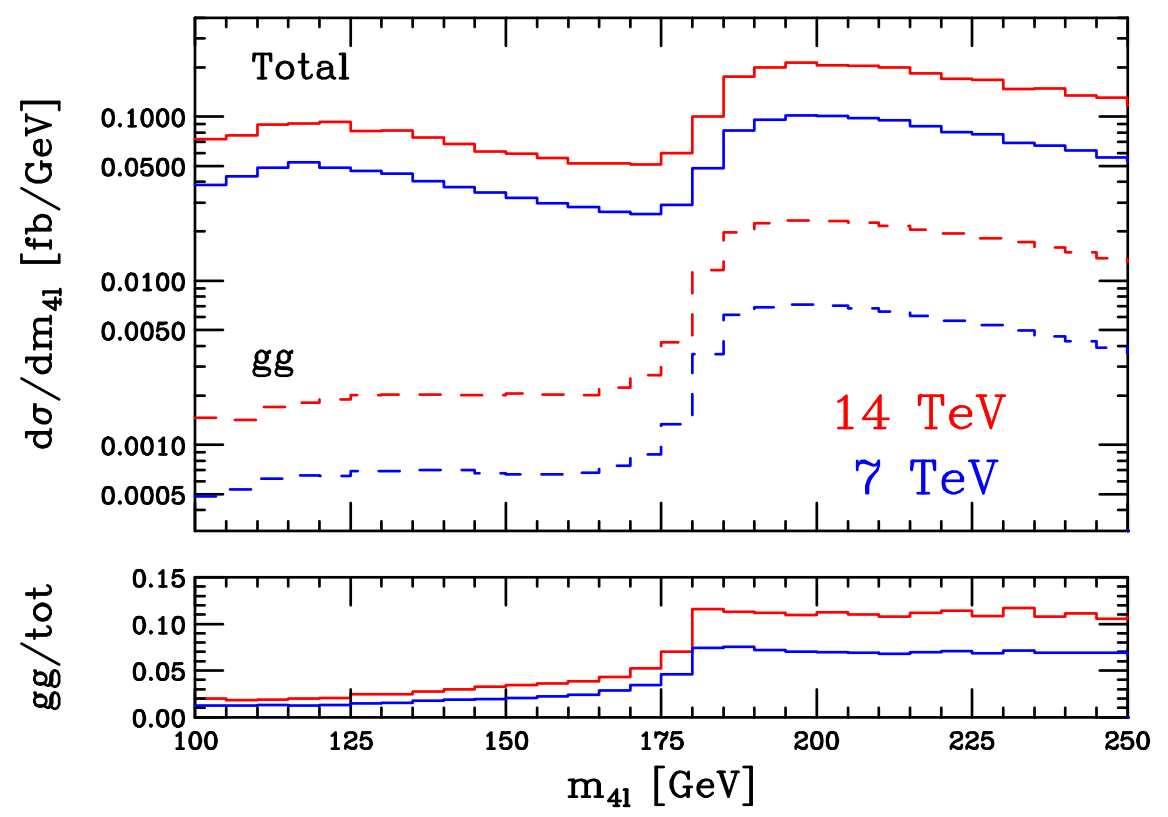

Figure 12. The invariant mass of the four lepton system in $Z / \gamma^{\star}\left(\rightarrow e^{+} e^{-}\right) Z / \gamma^{\star}\left(\rightarrow \mu^{+} \mu^{-}\right)$production at $\sqrt{s}=7$ and $\sqrt{s}=14 \mathrm{TeV}$, with the cuts of eq. (9.3). In the upper panel we show both the total NLO prediction (upper curves) and the contribution from the $g g$ initial state only (lower curves). In the lower panel we plot the fraction of the NLO prediction resulting from the $g g$ initial state.

revisited the treatment of many of the vector boson pair processes in order to ensure the relevance of the predictions for the LHC.

In order to enable simpler comparisons with experimental results we have implemented experimental photon isolation cuts into MCFM. This requires the inclusion of fragmentation contributions $[6,8]$, in which a QCD parton fragments into a photon plus hadronic energy. These fragmentation contributions require the introduction of fragmentation functions that contain both non-perturbative and perturbative information. Including this isolation condition extends the previous treatment of photons in MCFM, for which the smooth cone isolation of Frixione [9] had been used. Although this latter method is simple to implement theoretically it is not well-suited to experimental studies.

At the LHC, contributions to diboson production which proceed through a gluon initiated quark loop can have a significant effect on cross-sections. Although formally in perturbation theory they enter at NNLO the large flux of gluons at LHC center of mass energies can overcome the formal $\mathcal{O}\left(\alpha_{s}^{2}\right)$ suppression. Consequently we have included the gluon initiated processes $g g \rightarrow\{\gamma \gamma, Z \gamma, Z Z, W W\}$ whose contributions have been studied in the past [11-26]. We have also included higher order corrections to the $g g \rightarrow \gamma \gamma$ process $[13,14]$, which are formally at the level of $\mathrm{N}^{3} \mathrm{LO}$ in perturbation theory. These corrections are indeed of phenomenological relevance at the LHC, since at "NLO" the gluon contribution is around $20 \%$ of the total cross section at $\sqrt{s}=7 \mathrm{TeV}$.

We have presented detailed results for the diphoton process, $p p \rightarrow \gamma \gamma$ at the LHC, 
which is an extremely important channel for light Higgs boson searches. We have presented theoretical predictions for the $m_{\gamma \gamma}$ distribution using experimental cuts and isolation. We have illustrated how these cuts reduce the nominal cross section for a range of $\sqrt{s}$ appropriate to the LHC and also investigated the sensitivity of our predictions to the amount of hadronic energy in a fixed size isolation cone. We have also shown that experimental Higgs search cuts, which usually require staggered photon transverse momenta, produce large $K$-factors at NLO due to the limited kinematic configurations probed at leading order.

We also presented results for $W \gamma$ and $Z \gamma$ production. As a result of the new isolation procedures we were able to compare our NLO prediction for the cross sections with the recently measured values from CMS. We investigated the effects of various lepton cuts on cross-sections and predictions for distributions, particularly those cuts designed to suppress the contribution of photon radiation in the vector boson decay. This is an important consideration in the search for anomalous couplings between vector bosons and photons. Although the gluon-gluon contribution to the cross section is a few percent of the total at NLO, we found that as the minimum photon $p_{T}$ is increased the gluon initiated terms become relatively more important.

We studied the effects of including gluon initiated processes on $W W$ and $Z Z$ production. These pieces have been calculated using various methods in the past $[21,22,25,26]$, where it has been shown that the effects of the massive top quark are small. For this reason we have ignored the effect of a third generation (for $W W$ ) or top quark loop (for $Z Z$ ) and instead only include loops of massless quarks using the analytic formulae described in appendix B. For $Z Z$ production we also include the effects of singly-resonant diagrams that had previously been neglected in ref. [1]. For the case of $W W$ production we paid particular attention to the effects of a jet-veto on the NLO cross section, since at the LHC a jet-veto is necessary in order to reduce the abundant top background. We found that applying a jet-veto and Higgs search cuts increases the overall percentage of the cross section associated with the gluon initiated process. For $Z Z$ production we found that the contribution of the $g g$ process is only important in searches for a Higgs boson with a mass greater than $2 M_{Z}$. The fact that these $g g$ corrections can be large in some circumstances, suggests that the two loop corrections are worth calculating, to get a better idea of the associated theoretical error.

We also presented results for $W Z$ production at the LHC. As an example, we investigated the fraction of events that survive a minimum cut on the $Z$ transverse momentum. This quantity is important in regards to boosted searches for Higgs bosons and supersymmetry.

\section{Acknowledgments}

We thank Joe Lykken for useful discussions and Adrian Signer for providing us with a copy of the numerical program described in ref. [34]. We are grateful to Lance Dixon for comments on the first version of this manuscript. Fermilab is operated by Fermi Research Alliance, LLC under Contract No. DE-AC02-07CH11359 with the United States Department of Energy. 


\section{A Input parameters for phenomenological results}

The electroweak parameters that we regard as inputs are,

$$
\begin{array}{rlrl}
M_{W} & =80.398 \mathrm{GeV}, & M_{Z} & =91.1876 \mathrm{GeV}, \\
\Gamma_{W} & =2.1054 \mathrm{GeV}, & \Gamma_{Z} & =2.4952 \mathrm{GeV}, \\
G_{F} & =1.16639 \times 10^{-5} \mathrm{GeV}^{-2} . &
\end{array}
$$

Using the values of $M_{W}, M_{Z}$ and $G_{F}$ as above then determines $\alpha_{e . m .}\left(M_{Z}\right)$ and $\sin ^{2} \theta_{w}$ as outputs, where $\theta_{w}$ is the Weinberg angle. We find,

$$
\begin{aligned}
\sin ^{2} \theta_{w} & =1-M_{W}^{2} / M_{Z}^{2}=0.222646, \\
\alpha_{e . m .}\left(M_{Z}\right) & =\frac{\sqrt{2} G_{F} M_{W}^{2} \sin ^{2} \theta_{w}}{\pi}=\frac{1}{132.338} .
\end{aligned}
$$

This value of $\alpha_{e . m}$. may not correspond to the value of $\alpha_{e . m}$. used to fit the fragmentation functions in ref. [7]. The value of $\alpha_{e . m}$. used in their fit is hard to extract from ref. [7]. After isolation we believe that any potential mismatch will be of minor numerical significance.

\section{B Helicity amplitudes for gluon-gluon processes}

In this appendix we present results for three of the gluon initiated processes considered in the text, namely $g g \rightarrow Z \gamma, g g \rightarrow W W$ and $g g \rightarrow Z Z$. We first describe some general notation and then consider each of these processes in turn.

\section{B.1 Notation}

In order to specify the amplitudes we first introduce some notation. The QED and QCD couplings are denoted by $e$ and $g_{s}$ respectively and $Q^{q}$ is the charge of quark $q$ in units of $e$. The ratio of vector boson $V(=W, Z)$ and photon propagators is given by,

$$
\mathcal{P}_{V}(s)=\frac{s}{s-M_{V}^{2}+i \Gamma_{V} M_{V}},
$$

where $M_{V}$ and $\Gamma_{V}$ are the mass and width of the boson $V$. Fermions interact with the $Z$ boson through the following left- and right-handed couplings,

$$
\begin{aligned}
v_{L}^{e} & =\frac{-1+2 \sin ^{2} \theta_{w}}{\sin 2 \theta_{w}}, & v_{R}^{e} & =\frac{2 \sin ^{2} \theta_{w}}{\sin 2 \theta_{w}}, \\
v_{L}^{q} & =\frac{ \pm 1-2 Q^{q} \sin ^{2} \theta_{w}}{\sin 2 \theta_{w}}, & v_{R}^{q} & =-\frac{2 Q^{q} \sin ^{2} \theta_{w}}{\sin 2 \theta_{w}} .
\end{aligned}
$$

The subscripts $L$ and $R$ refer to whether the particle to which the $Z$ couples is left- or right-handed and the two signs in $v_{L}^{q}$ correspond to up $(+)$ and down (-) type quarks

We express the amplitudes in terms of spinor products defined as,

$$
\langle i j\rangle=\bar{u}_{-}\left(p_{i}\right) u_{+}\left(p_{j}\right), \quad[i j]=\bar{u}_{+}\left(p_{i}\right) u_{-}\left(p_{j}\right), \quad\langle i j\rangle[j i]=2 p_{i} \cdot p_{j} .
$$




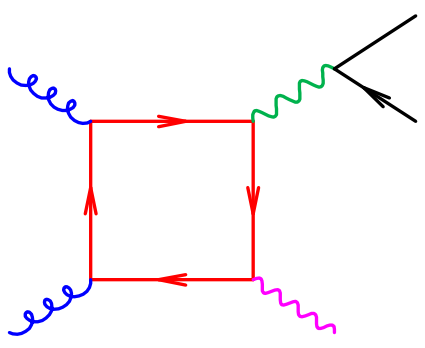

(a)

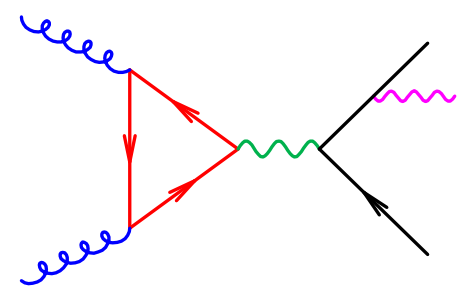

(b)

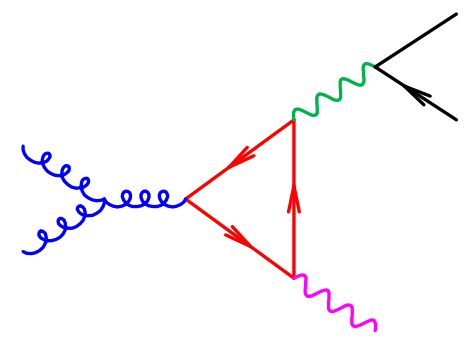

(c)

Figure 13. Examples of diagrams that could potentially contribute to $g g \rightarrow \gamma \ell \bar{\ell}$.

\section{B.2 Amplitudes for $g g \rightarrow Z \gamma$}

In this section we present results for the amplitudes relevant for the process,

$$
0 \rightarrow g\left(p_{1}\right)+g\left(p_{2}\right)+\gamma\left(p_{3}\right)+\ell\left(p_{4}\right)+\bar{\ell}\left(p_{5}\right)
$$

These amplitudes can be extracted from the fermion loop amplitudes $A_{6}^{v, a x}\left(1_{q}, 2_{\bar{q}}, 3_{g}, 4_{g}, 5_{\bar{\ell}}, 6_{\ell}\right)$ in ref. [27], by taking the limit in which the quark and antiquark are collinear, and renaming the momenta. The boxes and triangles which potentially could contribute to this amplitude are shown in figure 13. Considering the triangle diagrams first, we see that diagrams like figure 13(c) can never give a contribution because of colour. figure 13(b) with the vector coupling of the $Z$ to the fermion loop vanishes because of Furry's theorem, and with the axial coupling to the fermion loop vanishes because of Bose statistics (Landau-Yang theorem). We therefore only have to consider box diagrams like figure 13(a). In this case the diagrams with an axial coupling vanish, so we only have to consider box diagrams with a vector coupling.

The result for the fully dressed amplitude is,

$$
\begin{aligned}
\mathcal{A}_{5}^{1-\text { loop }}\left(1_{g}, 2_{g}, 3_{\gamma}, 4_{\ell}, 5_{\bar{\ell}}\right)=2 \sqrt{2} e^{3} \frac{g_{s}^{2}}{16 \pi^{2}} \delta^{a_{1} a_{2}} \\
\times \sum_{i=1}^{n_{f}} Q^{i}\left[-Q^{i}+\frac{1}{2} v_{L, R}^{e}\left(v_{L}^{i}+v_{R}^{i}\right) \mathcal{P}_{Z}\left(s_{56}\right)\right] A^{v}\left(1_{g}, 2_{g}, 3_{\gamma}, 4_{\ell}, 5_{\bar{\ell}}\right),
\end{aligned}
$$

where $a_{1}, a_{2}$ are the colour labels of the two gluons and there are $n_{f}$ flavours of massless quarks circulating in the loops. 
The amplitude $A_{5}^{(v)}\left(1_{g}^{+}, 2_{g}^{+}, 3_{\gamma}^{+}, 4_{\ell}^{-}, 5_{\bar{\ell}}^{+}\right)$is entirely rational and given by the following expression,

$$
A_{5}^{v}\left(1_{g}^{+}, 2_{g}^{+}, 3_{\gamma}^{+}, 4_{\ell}^{-}, 5_{\bar{\ell}}^{+}\right)=2\left\{\left[\frac{\langle 14\rangle^{2}[31]}{\langle 12\rangle^{2}\langle 13\rangle\langle 45\rangle}-\frac{1}{2} \frac{[53]^{2}}{\langle 12\rangle^{2}[54]}\right]+[1 \leftrightarrow 2]\right\} .
$$

The amplitude $A_{5}^{(v)}\left(1_{g}^{+}, 2_{g}^{+}, 3_{\gamma}^{-}, 4_{\ell}^{-}, 5_{\bar{\ell}}^{+}\right)$contains dependence on the box and triangle functions $\mathrm{L}_{0}(r), \mathrm{L}_{1}(r)$ and $\mathrm{Ls}_{-1}\left(r_{1}, r_{2}\right)$ that will be defined below. The result is,

$$
\begin{aligned}
A_{5}^{(v)}\left(1_{g}^{+}, 2_{g}^{+}, 3_{\gamma}^{-}\right. & \left., 4_{\ell}^{-}, 5_{\bar{\ell}}^{+}\right)=+2\left\{\frac{\langle 13\rangle^{2}\langle 24\rangle^{2}+\langle 14\rangle^{2}\langle 23\rangle^{2}}{\langle 12\rangle^{4}\langle 45\rangle} \mathrm{Ls}_{-1}\left(\frac{-s_{13}}{-s_{123}}, \frac{-s_{23}}{-s_{123}}\right)\right. \\
+ & {\left[2 \frac{\langle 23\rangle\langle 14\rangle\langle 24\rangle[21]}{[31]\langle 12\rangle^{3}\langle 45\rangle} \mathrm{L}_{0}\left(\frac{-s_{123}}{-s_{13}}\right)-\frac{\langle 24\rangle^{2}[21]^{2}}{[31]^{2}\langle 12\rangle^{2}\langle 45\rangle} \mathrm{L}_{1}\left(\frac{-s_{123}}{-s_{13}}\right)\right.} \\
& \left.\left.-\frac{\langle 13\rangle\langle 24\rangle[21][51]}{[31]\langle 12\rangle^{2}\langle 45\rangle[54]}\right]+[1 \leftrightarrow 2]\right\}
\end{aligned}
$$

We note that the $1 \leftrightarrow 2$ symmetry in this equation is to be applied to the terms inside square brackets only.

The final helicity amplitude $A_{5}^{(v)}\left(1_{g}^{+}, 2_{g}^{-}, 3_{\gamma}^{-}, 4_{\ell}^{-}, 5_{\bar{\ell}}^{+}\right)$can be obtained by exchange from eq. (B.7),

$$
\begin{aligned}
A_{5}^{(v)}\left(1^{+}, 2^{-}, 3_{\gamma}^{-}\right. & \left., 4_{\ell}^{-}, 5_{\bar{\ell}}^{+}\right)=-2\left\{\frac{[31]^{2}[25]^{2}+[35]^{2}[21]^{2}}{[32]^{4}[54]} \mathrm{Ls}_{-1}\left(\frac{-s_{13}}{-s_{123}}, \frac{-s_{12}}{-s_{123}}\right)\right. \\
+ & {\left[2 \frac{[21][35][25]\langle 23\rangle}{\langle 13\rangle[32]^{3}[54]} \mathrm{L}_{0}\left(\frac{-s_{123}}{-s_{13}}\right)-\frac{[25]^{2}\langle 23\rangle^{2}}{\langle 13\rangle^{2}[32]^{2}[54]} \mathrm{L}_{1}\left(\frac{-s_{123}}{-s_{13}}\right)\right.} \\
& \left.\left.-\frac{[31][25]\langle 23\rangle\langle 43\rangle}{\langle 13\rangle[32]^{2}[54]\langle 45\rangle}\right]+[2 \leftrightarrow 3]\right\},
\end{aligned}
$$

where again the $2 \leftrightarrow 3$ is to be applied to the terms inside square brackets only.

The latter two amplitudes are defined in terms of the following functions that arise from box integrals with one non-lightlike external line,

$$
\begin{aligned}
\mathrm{L}_{0}(r) & =\frac{\ln (r)}{1-r}, \\
\mathrm{~L}_{1}(r) & =\frac{\mathrm{L}_{0}(r)+1}{1-r}, \\
\mathrm{Ls}_{-1}\left(r_{1}, r_{2}\right) & =\mathrm{Li}_{2}\left(1-r_{1}\right)+\mathrm{Li}_{2}\left(1-r_{2}\right)+\ln r_{1} \ln r_{2}-\frac{\pi^{2}}{6},
\end{aligned}
$$

and the dilogarithm is defined by,

$$
\mathrm{Li}_{2}(x)=-\int_{0}^{x} d y \frac{\ln (1-y)}{y} .
$$

The remaining amplitudes can be obtained from these ones by simple symmetry operations. 

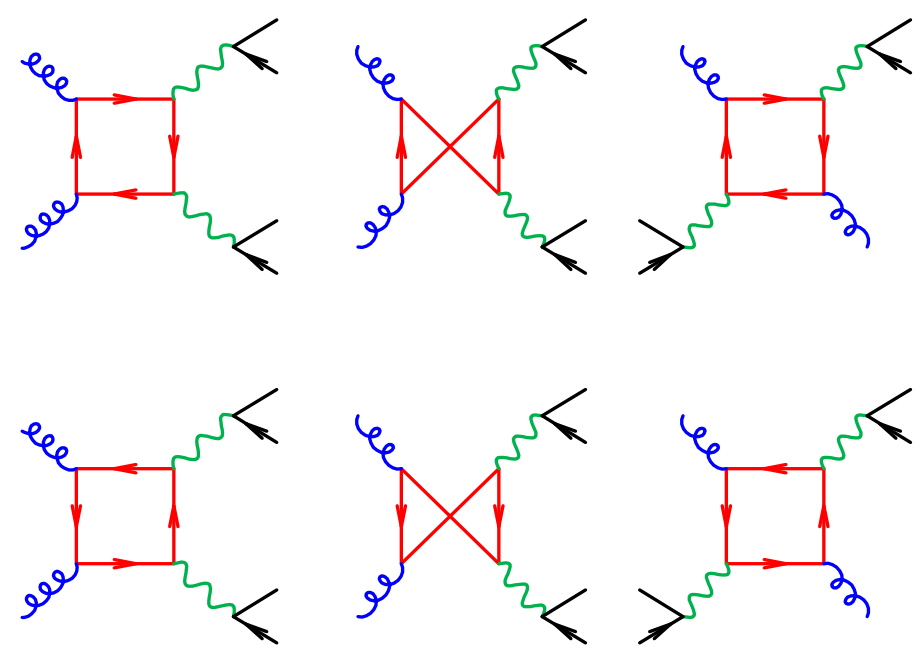

Figure 14. Diagrams that contribute to $g g \rightarrow W W$.

\section{B.3 Amplitudes for $g g \rightarrow W W$}

In this section we present results for the amplitudes relevant for the process,

$$
0 \rightarrow g\left(p_{1}\right)+g\left(p_{2}\right)+\nu_{\ell}\left(p_{3}\right)+\bar{\ell}\left(p_{4}\right)+\ell^{\prime}\left(p_{5}\right)+\bar{\nu}_{\ell^{\prime}}\left(p_{6}\right) .
$$

There are six contributing Feynman diagrams depicted in figure 14. These diagrams represent exactly the same set that appears in the calculation of certain contributions to the process $e^{+} e^{-} \rightarrow 4$ partons. We therefore simply reinterpret the compact expressions for such amplitudes presented in ref. [27], modifying the overall factor appropriately. Specifically, we find that the contribution from a single generation of massless quarks in the loop is given by,

$$
\begin{aligned}
\mathcal{A}_{6}^{1-\text { loop }} & \left(1_{g}^{h_{1}}, 2_{g}^{h_{2}}, 3_{\nu_{\ell}}^{-}, 4_{\bar{\ell}}^{+}, 5_{\ell^{\prime}}^{-}, 6_{\bar{\nu}_{\ell^{\prime}}^{+}}^{+}\right) \\
= & \delta^{a_{1} a_{2}}\left(\frac{g_{w}^{4} g_{s}^{2}}{16 \pi^{2}}\right) \mathcal{P}_{W}\left(s_{34}\right) \mathcal{P}_{W}\left(s_{56}\right) A_{6 ; 4}^{v}\left(4_{q}^{+}, 3_{\bar{q}}^{-}, 1_{g}^{h_{1}}, 2_{g}^{h_{2}} ; 5_{\bar{e}}^{-}, 6_{e}^{+}\right) .
\end{aligned}
$$

The helicities and colour labels of the two gluons are $h_{1}, h_{2}$ and $a_{1}, a_{2}$ respectively and the amplitude $A_{6 ; 4}^{v}$ is defined in Sections 2 and 11 of ref. [27]. The particle labelling on the right hand side of this equation is as written in ref. [27]. For our purposes we make the identification on the left hand side, $\left(q \rightarrow \bar{\ell}, \bar{q} \rightarrow \nu_{\ell}, \bar{e} \rightarrow \ell^{\prime}\right.$ and $\left.e \rightarrow \bar{\nu}_{\ell^{\prime}}\right)$.

\section{B.4 Amplitudes for $g g \rightarrow Z Z$}

In this section we present results for the amplitudes relevant for the process,

$$
0 \rightarrow g\left(p_{1}\right)+g\left(p_{2}\right)+\ell\left(p_{3}\right)+\bar{\ell}\left(p_{4}\right)+\ell^{\prime}\left(p_{5}\right)+\overline{\ell^{\prime}}\left(p_{6}\right) .
$$

The extension of the procedure outlined above for $g g \rightarrow W W$ is clear. One must now simply sum over all four possible helicity combinations for the leptonic decays. The result 
for the fully dressed amplitude is, for a particular choice of lepton helicities,

$$
\begin{aligned}
& \mathcal{A}_{6}^{1-\text { loop }}\left(1_{g}^{h_{1}}, 2_{g}^{h_{2}}, 3_{\ell}^{-}, 4_{\bar{\ell}}^{+}, 5_{\ell^{\prime}}^{-}, 6_{\ell^{\prime}}^{+}\right) \\
& =\delta^{a_{1} a_{2}} \frac{e^{4} g_{s}^{2}}{2 \pi^{2}} \sum_{i=1}^{n_{f}}\left[\left(-Q^{i}+\frac{1}{2} v_{L, R}^{\ell}\left(v_{L}^{i}+v_{R}^{i}\right) \mathcal{P}_{Z}\left(s_{34}\right)\right)\left(-Q^{i}+\frac{1}{2} v_{L, R}^{\ell^{\prime}}\left(v_{L}^{i}+v_{R}^{i}\right) \mathcal{P}_{Z}\left(s_{56}\right)\right)\right. \\
& \left.\quad+\frac{1}{4} v_{L, R}^{\ell} v_{L, R}^{\ell^{\prime}}\left(v_{L}^{i}-v_{R}^{i}\right)^{2} \mathcal{P}_{Z}\left(s_{34}\right) \mathcal{P}_{Z}\left(s_{56}\right)\right] A_{6 ; 4}^{v}\left(4_{q}^{+}, 3_{\bar{q}}^{-}, 1_{g}^{h_{1}}, 2_{g}^{h_{2}} ; 5_{\bar{e}}^{-}, 6_{e}^{+}\right) .
\end{aligned}
$$

The particle labelling on the right hand side of this equation is as written in ref. [27]. For our purposes we make the identification on the left hand side, $\left(q \rightarrow \bar{\ell}, \bar{q} \rightarrow \ell, \bar{e} \rightarrow \ell^{\prime}\right.$ and $e \rightarrow \overline{\ell^{\prime}}$.

Open Access. This article is distributed under the terms of the Creative Commons Attribution Noncommercial License which permits any noncommercial use, distribution, and reproduction in any medium, provided the original author(s) and source are credited.

\section{References}

[1] J.M. Campbell and R.K. Ellis, An update on vector boson pair production at hadron colliders, Phys. Rev. D 60 (1999) 113006 [hep-ph/9905386] [SPIRES].

[2] J.D. Hobbs, M.S. Neubauer and S. Willenbrock, Tests of the standard electroweak model at the energy frontier, arXiv: 1003.5733 [SPIRES].

[3] CMS collaboration, Observation of $W \gamma$ and $Z \gamma$ production at sqrts $=7 \mathrm{TeV}$, in preparation, CMS-EWK-10-008 (2011).

[4] ATLAS collaboration, G. Aad et al., Measurement of the $W W$ cross section in $\sqrt{s}=7 \mathrm{TeV}$ pp collisions with ATLAS, arXiv:1104.5225 [SPIRES].

[5] CMS collaboration, S. Chatrchyan et al., Measurement of $W W$ production and search for the Higgs boson in pp collisions at $\sqrt{s}=7$ TeV, Phys. Lett. B 699 (2011) 25 [arXiv:1102.5429] [SPIRES].

[6] S. Catani, M. Fontannaz, J.P. Guillet and E. Pilon, Cross-section of isolated prompt photons in hadron hadron collisions, JHEP 05 (2002) 028 [hep-ph/0204023] [SPIRES].

[7] L. Bourhis, M. Fontannaz and J.P. Guillet, Quark and gluon fragmentation functions into photons, Eur. Phys. J. C 2 (1998) 529 [hep-ph/9704447] [SPIRES].

[8] S. Catani and M.H. Seymour, A general algorithm for calculating jet cross sections in NLO QCD, Nucl. Phys. B 485 (1997) 291 [hep-ph/9605323] [SPIRES].

[9] S. Frixione, Isolated photons in perturbative QCD, Phys. Lett. B 429 (1998) 369 [hep-ph/9801442] [SPIRES].

[10] A.D. Martin, W.J. Stirling, R.S. Thorne and G. Watt, Parton distributions for the LHC, Eur. Phys. J. C 63 (2009) 189 [arXiv:0901.0002] [SPIRES].

[11] D.A. Dicus and S.S.D. Willenbrock, Photon pair production and the intermediate mass Higgs boson, Phys. Rev. D 37 (1988) 1801 [SPIRES].

[12] P.M. Nadolsky, C. Balázs, E.L. Berger and C.P. Yuan, Gluon-gluon contributions to the production of continuum diphoton pairs at hadron colliders, Phys. Rev. D 76 (2007) 013008 [hep-ph/0702003] [SPIRES]. 
[13] Z. Bern, A. De Freitas and L.J. Dixon, Two-loop amplitudes for gluon fusion into two photons, JHEP 09 (2001) 037 [hep-ph/0109078] [SPIRES].

[14] Z. Bern, L.J. Dixon and C. Schmidt, Isolating a light Higgs boson from the di-photon background at the LHC, Phys. Rev. D 66 (2002) 074018 [hep-ph/0206194] [SPIRES].

[15] L. Ametller, E. Gava, N. Paver and D. Treleani, Role of the QCD induced gluon-gluon coupling to gauge boson pairs in the multi-TeV region, Phys. Rev. D 32 (1985) 1699 [SPIRES].

[16] J.J. van der Bij and E.W.N. Glover, Photon Z boson pair production via gluon fusion, Phys. Lett. B 206 (1988) 701 [SPIRES].

[17] K.L. Adamson, D. de Florian and A. Signer, Gluon induced contributions to $Z \gamma$ production at hadron colliders, Phys. Rev. D 67 (2003) 034016 [hep-ph/0211295] [SPIRES].

[18] D.A. Dicus, C. Kao and W.W. Repko, Gluon production of gauge bosons, Phys. Rev. D 36 (1987) 1570 [SPIRES].

[19] E.W.N. Glover and J.J. van der Bij, Vector boson pair production via gluon fusion, Phys. Lett. B 219 (1989) 488 [SPIRES].

[20] E.W.N. Glover and J.J. van der Bij, $Z$ boson pair production via gluon fusion, Nucl. Phys. B 321 (1989) 561 [SPIRES].

[21] T. Binoth, M. Ciccolini, N. Kauer and M. Krämer, Gluon-induced WW background to Higgs boson searches at the LHC, JHEP 03 (2005) 065 [hep-ph/0503094] [SPIRES].

[22] T. Binoth, M. Ciccolini, N. Kauer and M. Krämer, Gluon-induced W-boson pair production at the LHC, JHEP 12 (2006) 046 [hep-ph/0611170] [SPIRES].

[23] T. Matsuura and J.J. van der Bij, Characteristics of leptonic signals for $Z$ boson pairs at hadron colliders, Z. Phys. C 51 (1991) 259 [SPIRES].

[24] C. Zecher, T. Matsuura and J.J. van der Bij, Leptonic signals from off-shell Z boson pairs at hadron colliders, Z. Phys. C 64 (1994) 219 [hep-ph/9404295] [SPIRES].

[25] N.E. Adam et al., Higgs working group summary report, arXiv:0803.1154 [SPIRES].

[26] T. Binoth, N. Kauer and P. Mertsch, Gluon-induced $Q C D$ corrections to $p p \rightarrow Z Z \rightarrow \ell \bar{\ell} \ell^{\prime} \bar{\ell}^{\prime}$, arXiv:0807.0024 [SPIRES].

[27] Z. Bern, L.J. Dixon and D.A. Kosower, One-loop amplitudes for $e^{+} e^{-}$to four partons, Nucl. Phys. B 513 (1998) 3 [hep-ph/9708239] [SPIRES].

[28] P. Aurenche, A. Douiri, R. Baier, M. Fontannaz and D. Schiff, Large $p_{T}$ double photon production in hadronic collisions: beyond leading logarithm QCD calculation, Z. Phys. C 29 (1985) 459 [SPIRES].

[29] T. Binoth, J.P. Guillet, E. Pilon and M. Werlen, A full next-to-leading order study of direct photon pair production in hadronic collisions, Eur. Phys. J. C 16 (2000) 311 [hep-ph/9911340] [SPIRES].

[30] ATLAS collaboration, Search for the Higgs boson in the diphoton final state with $38 \mathrm{pb}^{-1}$ of data recorded by the ATLAS detector in proton-proton collisions at sqrts $=7 \mathrm{TeV}$, ATLAS-CONF-2011-025 (2001).

[31] S. Frixione and G. Ridolfi, Jet photoproduction at HERA, Nucl. Phys. B507 (1997) 315 [hep-ph/9707345] [SPIRES]

[32] R.W. Brown, D. Sahdev and K.O. Mikaelian, $W^{ \pm} Z^{0}$ and $W^{ \pm} \gamma$ pair production in neutrino $e, p p$ and $\bar{p} p$ collisions, Phys. Rev. D 20 (1979) 1164 [SPIRES].

[33] J. Ohnemus, Order $\alpha_{s}$ calculations of hadronic $W^{ \pm} \gamma$ and $Z \gamma$ production, Phys. Rev. D 47 (1993) 940 [SPIRES]. 
[34] D. De Florian and A. Signer, $W \gamma$ and $Z \gamma$ production at hadron colliders, Eur. Phys. J. C 16 (2000) 105 [hep-ph/0002138] [SPIRES].

[35] L.J. Dixon, Z. Kunszt and A. Signer, Helicity amplitudes for $O\left(\alpha_{s}\right)$ production of $W^{+} W^{-}, W^{ \pm} Z, Z Z, W^{ \pm} \gamma$, or $Z \gamma$ pairs at hadron colliders, Nucl. Phys. B 531 (1998) 3 [hep-ph/9803250] [SPIRES].

[36] E. Accomando, A. Denner and C. Meier, Electroweak corrections to $W \gamma$ and $Z \gamma$ production at the LHC, Eur. Phys. J. C 47 (2006) 125 [hep-ph/0509234] [SPIRES].

[37] F. Campanario, C. Englert, M. Spannowsky and D. Zeppenfeld, NLO-QCD corrections to Wrj production, Europhys. Lett. 88 (2009) 11001 [arXiv:0908.1638] [SPIRES].

[38] K.O. Mikaelian, M.A. Samuel and D. Sahdev, The magnetic moment of weak bosons

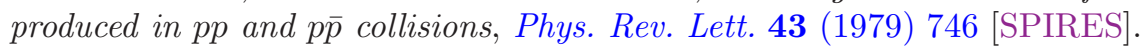

[39] U. Baur, T. Han and J. Ohnemus, QCD corrections and anomalous couplings in $Z \gamma$ production at hadron colliders, Phys. Rev. D 57 (1998) 2823 [hep-ph/9710416] [SPIRES].

[40] W. Hollik and C. Meier, Electroweak corrections to $\gamma Z$ production at hadron colliders, Phys. Lett. B 590 (2004) 69 [hep-ph/0402281] [SPIRES].

[41] R.W. Brown and K.O. Mikaelian, $W^{+} W^{-}$and $Z^{0} Z^{0}$ pair production in $e^{+} e^{-}, p p, p \bar{p}$ colliding beams, Phys. Rev. D 19 (1979) 922 [SPIRES].

[42] J. Ohnemus, An order $\alpha_{s}$ calculation of hadronic $W^{-} W^{+}$production, Phys. Rev. D 44 (1991) 1403 [SPIRES].

[43] S. Frixione, A next-to-leading order calculation of the cross-section for the production of $W^{+} W^{-}$pairs in hadronic collisions, Nucl. Phys. B 410 (1993) 280 [SPIRES].

[44] J. Ohnemus, Hadronic $Z Z, W^{-} W^{+}$and $W^{ \pm} Z$ production with $Q C D$ corrections and leptonic decays, Phys. Rev. D 50 (1994) 1931 [hep-ph/9403331] [SPIRES].

[45] L.J. Dixon, Z. Kunszt and A. Signer, Vector boson pair production in hadronic collisions at order $\alpha_{s}$ : lepton correlations and anomalous couplings, Phys. Rev. D 60 (1999) 114037 [hep-ph/9907305] [SPIRES].

[46] J.M. Campbell, R.K. Ellis and G. Zanderighi, Next-to-leading order predictions for $W W+$ jet distributions at the LHC, JHEP 12 (2007) 056 [arXiv:0710.1832] [SPIRES].

[47] S. Dittmaier, S. Kallweit and P. Uwer, $N L O Q C D$ corrections to $p p / p \bar{p} \rightarrow W W+j e t+X$ including leptonic $W$-boson decays, Nucl. Phys. B 826 (2010) 18 [arXiv:0908.4124] [SPIRES].

[48] T. Melia, K. Melnikov, R. Rontsch and G. Zanderighi, Next-to-leading order QCD predictions for $W+W+j j$ production at the LHC, JHEP 12 (2010) 053 [arXiv:1007.5313] [SPIRES].

[49] T. Melia, K. Melnikov, R. Rontsch and G. Zanderighi, $N L O$ QCD corrections for $W^{+} W^{-}$ pair production in association with two jets at hadron colliders, arXiv:1104.2327 [SPIRES].

[50] J. Ohnemus, An order $\alpha_{s}$ calculation of hadronic $W^{ \pm} Z$ production, Phys. Rev. D 44 (1991) 3477 [SPIRES].

[51] S. Frixione, P. Nason and G. Ridolfi, Strong corrections to $W Z$ production at hadron colliders, Nucl. Phys. B 383 (1992) 3 [SPIRES].

[52] F. Campanario, C. Englert, S. Kallweit, M. Spannowsky and D. Zeppenfeld, NLO QCD corrections to $W Z+$ jet production with leptonic decays, JHEP 07 (2010) 076 [arXiv: 1006.0390] [SPIRES].

[53] D0 collaboration, V.M. Abazov et al., Measurement of the $W Z \rightarrow \ell \nu \ell \ell$ cross section and limits on anomalous triple gauge couplings in pp collisions at $\sqrt{s}=1.96 \mathrm{TeV}$, Phys. Lett. B 695 (2011) 67 [arXiv:1006.0761] [SPIRES]. 
[54] The ATLAS collaboration, G. Aad et al., Expected performance of the ATLAS experiment - Detector, trigger and physics, arXiv:0901.0512 [SPIRES].

[55] CMS collaboration, G.L. Bayatian et al., CMS technical design report, volume II: physics performance, J. Phys. G 34 (2007) 995 [SPIRES].

[56] J.M. Butterworth, A.R. Davison, M. Rubin and G.P. Salam, Jet substructure as a new Higgs search channel at the LHC, Phys. Rev. Lett. 100 (2008) 242001 [arXiv:0802.2470] [SPIRES].

[57] A. Abdesselam et al., Boosted objects: a probe of beyond the standard model physics, Eur. Phys. J. C 71 (2011) 1661 [arXiv:1012.5412] [SPIRES].

[58] J. Ohnemus and J.F. Owens, An order $\alpha_{s}$ calculation of hadronic $Z Z$ production, Phys. Rev. D 43 (1991) 3626 [SPIRES].

[59] B. Mele, P. Nason and G. Ridolfi, QCD radiative corrections to $Z$ boson pair production in hadronic collisions, Nucl. Phys. B 357 (1991) 409 [SPIRES].

[60] T. Binoth, T. Gleisberg, S. Karg, N. Kauer and G. Sanguinetti, NLO QCD corrections to $Z Z+$ jet production at hadron colliders, Phys. Lett. B 683 (2010) 154 [arXiv:0911.3181] [SPIRES]. 